\title{
Dense gas in a giant molecular filament ${ }^{\star}$
}

\author{
Y. Wang $(\text { 王渊 })^{1}$, H. Beuther ${ }^{1}$, N. Schneider ${ }^{2}$, S. E. Meidt ${ }^{1}$, H. Linz ${ }^{1}$, S. Ragan ${ }^{3}$, C. Zucker ${ }^{4}$, C. Battersby ${ }^{5}$, J. D. Soler ${ }^{1}$, \\ E. Schinnerer ${ }^{1}$, F. Bigiel ${ }^{6}$, D. Colombo ${ }^{7}$, and Th. Henning ${ }^{1}$
}

\author{
${ }^{1}$ Max-Planck-Insitute for Astronomy, Königstuhl 17, 69117 Heidelberg \\ e-mail: wang@mpia.de \\ 2 I. Physik. Institut, University of Cologne, Zülpicher Str.77, 50937 Cologne, Germany \\ ${ }^{3}$ School of Physics and Astronomy, Cardiff University, Queenâs Buildings, The Parade, Cardiff, CF24 3AA, UK \\ ${ }^{4}$ Harvard Astronomy, Harvard-Smithsonian Center for Astrophysics, 60 Garden Street, Cambridge, MA 02138, USA \\ ${ }^{5}$ Department of Physics, University of Connecticut, Storrs, CT 06269, USA \\ ${ }^{6}$ Argelander Institut für Astronomie, Auf dem Hügel 71, 53121 Bonn, Germany \\ ${ }^{7}$ Max-Planck-Institut für Radioastronomie, Auf dem Hügel 69, 53121 Bonn, Germany
}

Received 11 March 2020 / Accepted 22 June 2020

\begin{abstract}
Context. Recent surveys of the Galactic plane in the dust continuum and CO emission lines reveal that large ( $\gtrsim 50 \mathrm{pc})$ and massive $\left(\gtrsim 10^{5} M_{\odot}\right)$ filaments, know as giant molecular filaments (GMFs), may be linked to Galactic dynamics and trace the mid-plane of the gravitational potential in the Milky Way. Yet our physical understanding of GMFs is still poor.

Aims. We investigate the dense gas properties of one GMF, with the ultimate goal of connecting these dense gas tracers with star formation processes in the GMF.

Methods. We imaged one entire GMF located at $l \sim 52-54^{\circ}$ longitude, GMF54 ( $68 \mathrm{pc}$ long), in the empirical dense gas tracers using the $\mathrm{HCN}(1-0), \mathrm{HNC}(1-0)$, and $\mathrm{HCO}^{+}(1-0)$ lines, and their ${ }^{13} \mathrm{C}$ isotopologue transitions, as well as the $\mathrm{N}_{2} \mathrm{H}^{+}(1-0)$ line. We studied the dense gas distribution, the column density probability density functions (N-PDFs), and the line ratios within the GMF.

Results. The dense gas molecular transitions follow the extended structure of the filament with area filling factors between 0.06 and 0.28 with respect to ${ }^{13} \mathrm{CO}(1-0)$. We constructed the N-PDFs of $\mathrm{H}_{2}$ for each of the dense gas tracers based on their column densities and assumed uniform abundance. The N-PDFs of the dense gas tracers appear curved in log-log representation, and the $\mathrm{HCO}^{+} \mathrm{N}-\mathrm{PDF}$ has the flattest power-law slope index. Studying the N-PDFs for sub-regions of GMF54, we found an evolutionary trend in the N-PDFs that high-mass star-forming and photon-dominated regions have flatter power-law indices. The integrated intensity ratios of the molecular lines in GMF54 are comparable to those in nearby galaxies. In particular, the $\mathrm{N}_{2} \mathrm{H}^{+} /{ }^{13} \mathrm{CO}$ ratio, which traces the dense gas fraction, has similar values in GMF54 and all nearby galaxies except Ultraluminous Infrared Galaxies.

Conclusions. As the largest coherent cold gaseous structure in our Milky Way, GMFs, are outstanding candidates for connecting studies of star formation on Galactic and extragalactic scales. By analyzing a complete map of the dense gas in a GMF we have found that: (1) the dense gas N-PDFs appear flatter in more evolved regions and steeper in younger regions, and (2) its integrated dense gas intensity ratios are similar to those of nearby galaxies.
\end{abstract}

Key words. ISM: clouds - ISM: molecules - stars: formation - radio lines: ISM

\section{Introduction}

Studies of nearby molecular clouds (MCs) show that the dense regions of MCs are permeated with filaments that contain sites of star formation (e.g., André et al. 2014). Recent observations identified a class of large $(\gtrsim 50 \mathrm{pc})$ and massive $\left(\gtrsim 10^{5} M_{\odot}\right)$ filaments, known as giant molecular filaments (GMFs; Jackson et al. 2010; Goodman et al. 2014; Ragan et al. 2014; Zucker et al. 2015; Wang et al. 2015, 2016, 2020; Li et al. 2016; Abreu-Vicente et al. 2016). Many of these giant filaments lie along, or extremely close to spiral arms in the position-position-velocity space, suggesting they may trace the dense "spine" of the spiral arms and the mid-plane of the gravitational potential in the Milky Way (MW; Goodman et al. 2014; Zucker et al. 2015). Detailed intercomparison of a sample of long filaments from the literature suggests that there may be different classes of filaments in both

\footnotetext{
* Datacubes and integrated intensity maps are only available at the CDS via anonymous ftp to cdsarc.u-strasbg.fr (130.79.128.5) or via http://cdsarc.u-strasbg.fr/viz-bin/cat/J/A+A/641/ A53
}

their physical properties and their association with the Galactic structure (Zucker et al. 2018). Giant molecular filaments are the largest coherent cold gas structures in our Milky Way yet our physical understanding of GMFs is still poor, limited to estimates of their occurrence, gas masses, and lengths (Ragan et al. 2014; Abreu-Vicente et al. 2016; Zucker et al. 2018). Observations of nearby galaxies have resolved giant MCs with masses and sizes similar to those of GMFs (10 to 200 pc, e.g., Hughes et al. 2013; Schinnerer et al. 2013; Leroy et al. 2016; Li et al. 2016; Faesi et al. 2018; Herrera et al. 2020), suggesting that GMFs could be analogous to these extragalactic giant MCs and could connect studies of star formation on Galactic and extragalactic scales.

So far, GMF studies have relied on Galactic plane surveys, either in dust tracers like (sub)millimeter emission (e.g., Hi-GAL $^{1}$, Molinari et al. 2010; ATLASGAL ${ }^{2}$, Schuller et al. 2009) or in low to intermediate spatial resolution surveys of CO

The Herschel infrared Galactic Plane Survey.
2 The APEX Telescope Large Area Survey of the Galaxy. 


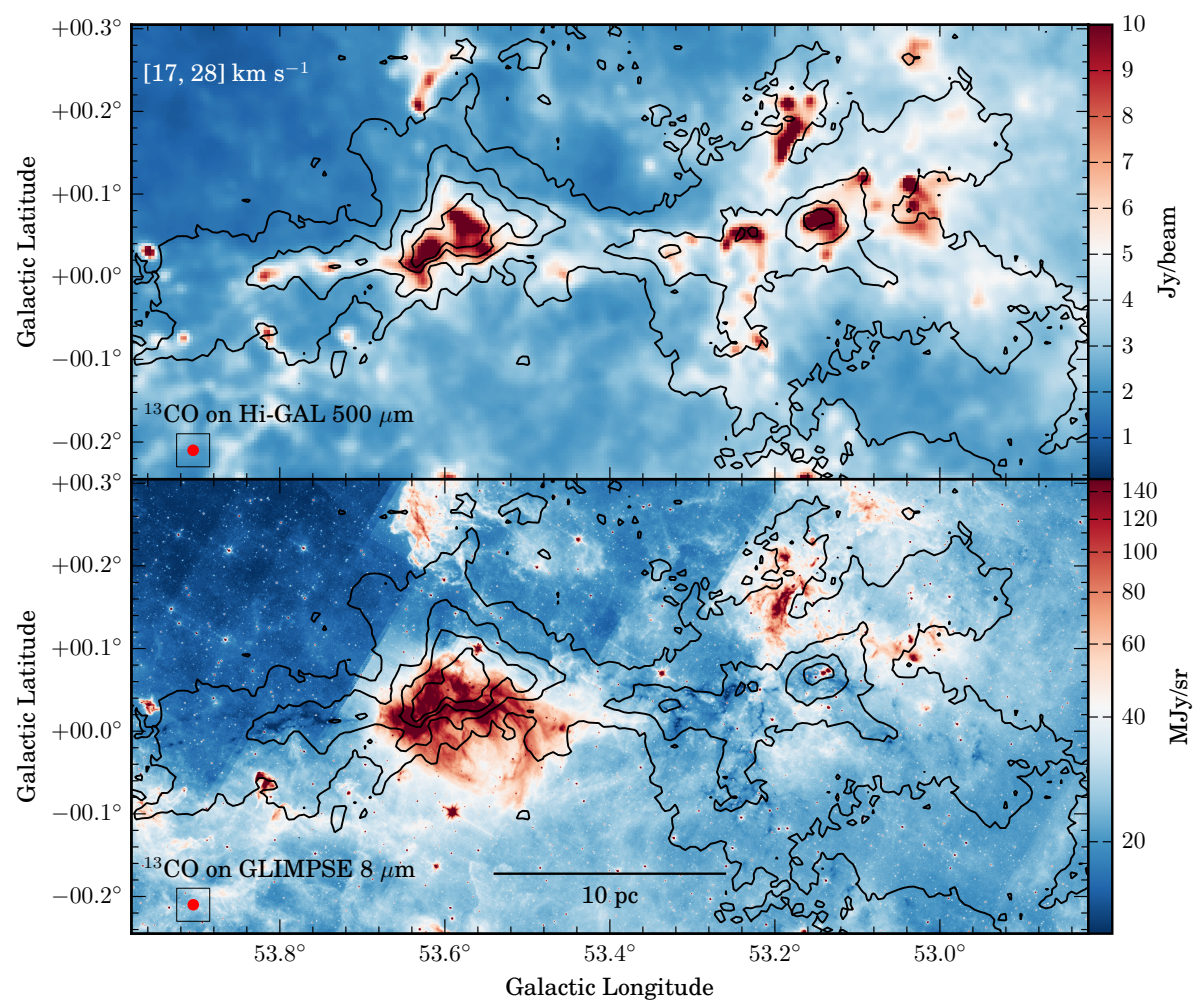

Fig. 1. Integrated intensity contours of the GRS ${ }^{13} \mathrm{CO}(1-0)$ emission (17-28 $\mathrm{km} \mathrm{s}^{-1}$, Jackson et al. 2006) overlaid on the Hi-GAL $500 \mu \mathrm{m}$ continuum map at an angular resolution of $36^{\prime \prime}$ (Molinari et al. 2010, top panel) and GLIMPSE $8 \mu \mathrm{m}$ map (Churchwell et al. 2009, bottom panel) toward the giant molecular filament GMF54. Contour levels are 5, 15, 25, and $35 \sigma$ $\left(\sigma=0.75 \mathrm{~K} \mathrm{~km} \mathrm{~s}^{-1}\right)$. The beam of the ${ }^{13} \mathrm{CO}(1-$ $0)$ emission is marked in the bottom left of each panel. and its isotopologues (e.g., GRS ${ }^{3}$, Jackson et al. 2006; COHRS ${ }^{4}$, Dempsey et al. 2013; SEDIGISM $^{5}$, Schuller et al. 2017). However, little in known about the high-volume-density gas that will eventually form stars.

To study this, we selected the giant molecular filament located at $l \sim 52-54 \mathrm{deg}$ longitude (GMF54, Fig. 1), which was identified by Ragan et al. (2014) in infrared extinction and confirmed using the GRS ${ }^{13} \mathrm{CO}(1-0)$ data (Jackson et al. 2006) as a velocity-coherent filament at a distance of $\sim 2 \mathrm{kpc}$. With the ${ }^{13} \mathrm{CO}$ data, Ragan et al. (2014) estimated the total length and mass of GMF54 to be $\sim 68 \mathrm{pc}$ and $6.8 \times 10^{4} M_{\odot}$, respectively.

As a common tool to study the physical properties of molecular clouds, column density probability density functions (N-PDFs) are widely used both in observational (e.g., Lombardi et al. 2008; Kainulainen et al. 2009; Alves de Oliveira et al. 2014; Sadavoy et al. 2014; Abreu-Vicente et al. 2015; Stutz \& Kainulainen 2015; Schneider et al. 2015a; Lin et al. 2017) and theoretical studies (e.g., Ostriker et al. 2001; Federrath et al. 2010; Federrath \& Klessen 2013; Burkhart et al. 2017; Chen et al. 2018; Körtgen et al. 2019). The shape of the N-PDF depends on the physical processes dominating the cloud and can thus be used to study the evolution of the molecular clouds. From simulations, the early evolutionary stage of a molecular cloud is dominated by turbulence and the N-PDF shows a log-normal shape. The width of the log-normal N-PDF is determined by the turbulent motions (see e.g., Federrath et al. 2010; BallesterosParedes et al. 2011; Kritsuk et al. 2011; Federrath \& Klessen 2013; Burkhart et al. 2015; Bialy et al. 2017; Körtgen et al. 2019). As the cloud evolves into a gravity-dominated system, the N-PDF develops a high column density power-law tail with a slope of around -2 (Klessen 2000; Girichidis et al. 2014).

\footnotetext{
3 The Galactic Ring Survey.

4 The CO High-Resolution Survey.

5 Structure, excitation, and dynamics of the inner Galactic interstellar medium.
}

Observations indicate that star-forming clouds show such tails, providing support for this scenario (e.g., Kainulainen et al. 2009; Schneider et al. 2013). Furthermore, the slope of the powerlaw N-PDF can be related to the evolutionary stages of the clouds as steeper slopes possibly indicate earlier quiescent stages (e.g., Kritsuk et al. 2011; Federrath \& Klessen 2013; Ward et al. 2014), while flatter slopes or a second flatter power-law tail, often found in high-mass star-forming regions may indicate feedback effects (Tremblin et al. 2014; Schneider et al. 2015b).

Column density probability density functions are often constructed using dust extinction or continuum emission observations (e.g., Lombardi et al. 2008; Kainulainen et al. 2009; Sadavoy et al. 2014; Stutz \& Kainulainen 2015; Schneider et al. 2015b). However, since GMFs lie close to the Galactic midplane, heavy contamination from foreground and background emission makes it very difficult to study N-PDFs with continuum emission as it traces the entire line of sight through the Milky Way disk. With the velocity information of molecular line observations, we can easily distinguish different clouds along the line of sight. So far, a few studies have investigated the molecular cloud properties with N-PDFs obtained from low J transitions of ${ }^{12} \mathrm{CO}$ or ${ }^{13} \mathrm{CO}$ observations (Goldsmith et al. 2008; Wong et al. 2008; Goodman et al. 2009; Carlhoff et al. 2013; Schneider et al. 2015b; Sun et al. 2020), which cannot trace the column density of dense cores due to high optical depth. Schneider et al. (2016) found that compared to dust N-PDF, $\mathrm{N}-\mathrm{PDF}$ of the dense gas tracers $\mathrm{N}_{2} \mathrm{H}^{+}(1-0)$ and $\mathrm{CS}(2-1)$ can recover the power-law tail well independent of the exact column density.

\section{Observation and data reduction}

We describe the observations and data reduction of different dataset used in the paper. 


\subsection{Dense gas tracers with IRAM $30 \mathrm{~m}$}

The giant filament GMF54 was observed with the IRAM $30 \mathrm{~m}$ telescope in September 2017 and November 2017 with the EMIR receiver and the Fourier Transform Spectrometer (FTS) backends at $3 \mathrm{~mm}$. The receiver was tuned to center at $91.4 \mathrm{GHz}$ with dual-polarization to cover the dense gas tracers $\mathrm{HCO}^{+}(1-0)$, $\mathrm{HCN}(1-0)$, $\mathrm{HNC}(1-0)$, and their ${ }^{13} \mathrm{C}$ isotopologues, including the cold and dense gas tracer $\mathrm{N}_{2} \mathrm{H}^{+}(1-0)$. The FTS backends were used to cover the $16 \mathrm{GHz}$ bandwidth of the receiver with a uniform spectral resolution of $200 \mathrm{kHz}$, which results in a velocity resolution of $\sim 0.7 \mathrm{~km} \mathrm{~s}^{-1}$ at $3 \mathrm{~mm}$. The observations were carried out in the on-the-fly (OTF) mode employing position switching to an OFF position sufficiently far away. The filament was scanned at least once along the Galactic longitude and latitude directions, respectively, in order to reduce scanning effects. The system temperature and other calibration parameters were measured every 10 to $15 \mathrm{~min}$. Jupiter and standard millimeter calibrators were observed regularly to calibrate the pointing and focus. During the observation, the weather conditions in general were good and the radiometer opacity $\tau$ at 225 was measured at around 0.5; two OTF maps observed on 9 September, however, had a precipitable water vapor (PWV) larger than $40 \mathrm{~mm}$, and the data were therefore dropped and the area re-observed later.

The spectra were calibrated with CLASS, which is part of the GILDAS software package ${ }^{6}$. We converted the data from antenna temperature $\left(T_{\mathrm{A}}\right)$ to main beam brightness temperature $\left(T_{\mathrm{mb}}\right)$ using a forward efficiency $\left(F_{\text {eff }}=95 \%\right.$ at $\left.3 \mathrm{~mm}\right)$ and main beam efficiency ( $B_{\text {eff }}=81 \%$ at $3 \mathrm{~mm}$ ) following equation $T_{\mathrm{mb}}=T_{\mathrm{A}} \times F_{\text {eff }} / B_{\text {eff. }}$. A linear function was fitted to the line free channels of the spectra to subtract the baseline. The final $3 \mathrm{~mm}$ data were all smoothed to a common spectral resolution of $0.7 \mathrm{~km} \mathrm{~s}^{-1}$ and a beam size of $32^{\prime \prime}(\sim 0.32 \mathrm{pc}$ at $2 \mathrm{kpc}$ distance), and the rms noise level of $T_{\mathrm{mb}}$ measured at line free channels is around $0.05 \mathrm{~K}$.

\section{2. ${ }^{13} \mathrm{CO}(1-0)$}

The ${ }^{13} \mathrm{CO}(1-0)$ data are from the Galactic Ring Survey (GRS, Jackson et al. 2006), with a spectral resolution of $0.21 \mathrm{~km} \mathrm{~s}^{-1}$ and a full width at half maximum (FWHM) beam size of $46^{\prime \prime}$. After converting the antenna temperature $\left(T_{\mathrm{A}}\right)$ to main beam brightness temperature $\left(T_{\mathrm{mb}}\right)$ using a main beam efficiency of 0.48 (Jackson et al. 2006), the line free channel rms noise level of $T_{\mathrm{mb}}$ is around $0.22 \mathrm{~K}$.

\subsection{Dust continuum data}

The dust temperature and column density maps were produced by Zucker et al. (2018) by performing pixel-by-pixel modified blackbody fits to the Hi-GAL 160, 250, 350, and $500 \mu \mathrm{m}$ continuum map using the HiGal_SEDfitter ${ }^{7}$ code (Wang et al. 2015 ) with a fixed dust opacity of $\beta=1.75$ and a gas-to-dust ratio of 100. To trace cold gas better, the $70 \mu \mathrm{m}$ band was excluded from the fitting due to its high optical depth toward very dense regions. We subtract a constant column density value $\left(3.46 \times 10^{21} \mathrm{~cm}^{-2}\right)$ measured from a $1^{\prime}$ radius circular area centered at $l=53.69^{\circ}$ and $b=-0.30^{\circ}$ to remove the line-of-sight contamination (Schneider et al. 2015b; Ossenkopf-Okada et al. 2016).

\footnotetext{
6 http://www.iram.fr/IRAMFR/GILDAS

7 https://github.com/keflavich/HiGal_SEDfitter
}

\section{Results}

We present the molecular line emission results, including the emission maps, column densities, and N-PDFs, in this section.

\subsection{Dense gas tracers with IRAM $30 \mathrm{~m}$}

While the ${ }^{13} \mathrm{CO}(1-0)$ map (Fig. 1) shows the extended nature of the giant filament GMF54, the integrated intensity maps of $\mathrm{HCO}^{+}, \mathrm{HCN}, \mathrm{HNC}$, and $\mathrm{N}_{2} \mathrm{H}^{+}$in Fig. 2 show that these dense gas spectral lines trace the extended structure of the filament although a much smaller area is detected compared to the ${ }^{13} \mathrm{CO}$ map. The integral velocity range for each tracer was determined to include all emissions associated with the filament. Since we want to include the emission of all hyper-fine structures, a larger integrated velocity range (as shown in Fig. 2) is adopted for $\mathrm{HCN}(1-0)$ and $\mathrm{N}_{2} \mathrm{H}^{+}(1-0)$, which also includes some emission at velocities $\gtrsim 35 \mathrm{~km} \mathrm{~s}^{-1}$ or $\lesssim 10 \mathrm{~km} \mathrm{~s}^{-1}$. These emissions that lie in regions marked with red ellipses in Fig. 2 are at different distances and are unrelated to the filament, so we exclude them from the following discussion. As the spectra in Fig. 2 show, the filament is dominated by one component centered at $20 \mathrm{~km} \mathrm{~s}^{-1}$. The contamination from the other two components (peaked at $\sim 5$ and $\sim 36 \mathrm{~km} \mathrm{~s}^{-1}$, respectively) is negligible.

To quantify how much extended structure is traced by different molecular lines, we calculated the area filling factor of the dense gas integrated intensity map relative to the ${ }^{13} \mathrm{CO}(1-0)$ map. We measured the area of each molecular line map where $S / N>3$, and divided it by the ${ }^{13} \mathrm{CO}$ map area to get the filling factor, and the results are listed in Table 1. Comparing to the ${ }^{13} \mathrm{CO}$ emission, these typical dense gas tracers cover between 6 and $28 \%$ of the area of the ${ }^{13} \mathrm{CO}$ emission, depending on the tracer (Table 1). If we smooth and regrid our $3 \mathrm{~mm}$ integrated intensity maps into the same angular resolution of $46^{\prime \prime}$ and pixel size of $22^{\prime \prime}$ as the ${ }^{13} \mathrm{CO}$ data, the filling factors of these dense gas tracers increases by $\sim 70 \%$ (see Table 1 ). The filling factors should be taken in to account for extragalactic studies.

We list the upper energy level, the critical density ( $\left.n_{\text {crit }}\right)$ under the simplifying assumptions of two-levels systems and optically thin emission, and the effective excitation density $\left(n_{\text {eff }}\right)$ of the lines we used in Table 2. Recent studies show that these molecular lines trace much lower densities than their critical densities (e.g., Kauffmann et al. 2017; Pety et al. 2017). Compared to $n_{\text {crit }}, n_{\text {eff }}$ gives a better estimate of the approximate density at which a modest $\left(1 \mathrm{~K} \mathrm{~km} \mathrm{~s}^{-1}\right)$ molecular line can be observed (Shirley 2015). Among these four dense gas tracers $\left(\mathrm{HCO}^{+}, \mathrm{HCN}, \mathrm{HNC}\right.$, and $\left.\mathrm{N}_{2} \mathrm{H}^{+}\right)$, we observe that $\mathrm{HCO}^{+}(1-0)$ has the smallest $n_{\text {eff }}$, and also traces the most extended structure, while $\mathrm{N}_{2} \mathrm{H}^{+}(1-0)$ has the highest $n_{\text {eff }}$ and traces as expected the most compact structure. On the other hand, $\mathrm{HCN}(1-0)$ has both a higher $n_{\text {eff }}$ and a higher $n_{\text {crit }}$ than HNC(1-0), yet traces a more extended structure than HNC(1-0). Kauffmann et al. (2017) also found that $\mathrm{HCN}(1-0)$ typically traces gas at a density of $\sim 870 \mathrm{~cm}^{-3}$, while $\mathrm{N}_{2} \mathrm{H}^{+}(1-0)$ traces a much higher density of $\sim 4 \times 10^{3} \mathrm{~cm}^{-3}$

The ratios of the molecular line integrated intensities are often used to trace different physical properties of the gas. The $\mathrm{HCN} / \mathrm{CO}$ integrated intensity ratio is commonly used to trace the dense gas fraction in galactic and extragalactic studies (e.g., Gao \& Solomon 2004; Lada et al. 2012), and the HCN/HNC ratio is considered to be able to trace the evolutionary stages of the molecular cloud (Schilke et al. 1992; Graninger et al. 2014; Hacar et al. 2020). To compare the intensity of different lines properly, we first integrated all four dense gas tracers and the ${ }^{13} \mathrm{CO}$ line 

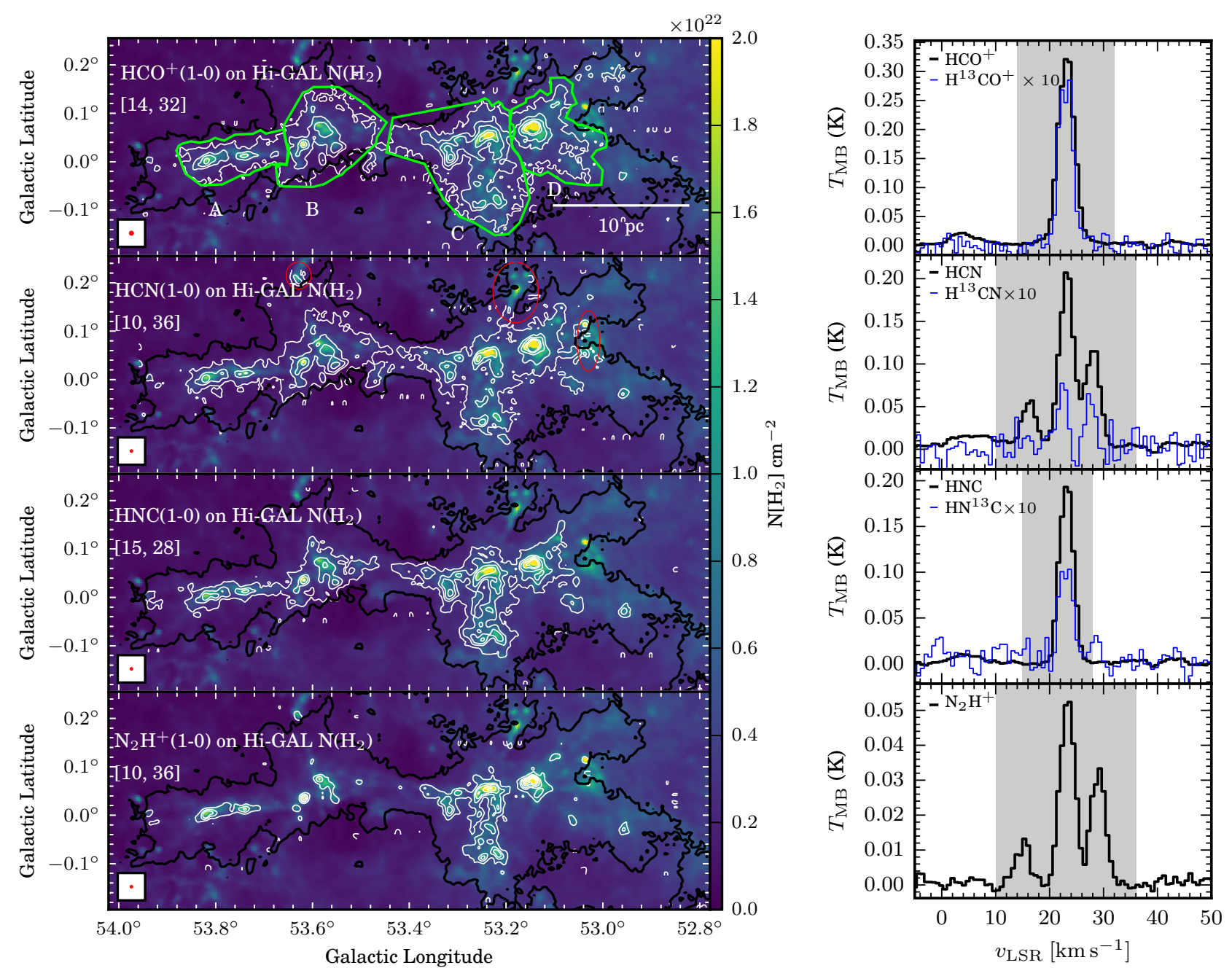

Fig. 2. Left panels: integrated intensity contours of the $\mathrm{HCO}^{+}(1-0), \mathrm{HCN}(1-0), \mathrm{HNC}(1-0)$, and $\mathrm{N}_{2} \mathrm{H}^{+}(1-0)$ overlaid on the molecular hydrogen column density map derived from fitting Hi-GAL 160, 250, 350, and $500 \mu \mathrm{m}$ continuum maps (Zucker et al. 2018). Contour levels are 3, 9, 15, 25 , and 35 times of the respective $\sigma$ of each integrated intensity map. The noise level of each panel from top to bottom: 0.28, 0.40, 0.22, and $0.29 \mathrm{~K} \mathrm{~km} \mathrm{~s}^{-1}$, respectively. The integral velocity range for each line is labeled in each panel in units of $\mathrm{km} \mathrm{s}^{-1}$. The polygons in the $\mathrm{HCO}^{+}(1-0)$ panel outline the four sub-regions, A, B, C, and D, which we studied in Sect. 3.3. The thick black contour in each panel traces the $5 \sigma$ level of the GRS ${ }^{13} \mathrm{CO}(1-0)$ integrated intensity. The red ellipses mark the emissions that are at a different distance and not associated with the filament. The beam of the molecular line emission (32") is marked in the bottom left of each panel. Right panels: average spectra of the dense gas tracers across the filament. The thick black spectra are $\mathrm{HCO}^{+}(1-0), \mathrm{HCN}(1-0), \mathrm{HNC}(1-0)$, and $\mathrm{N}_{2} \mathrm{H}^{+}(1-0)$ lines, respectively. The thin blue spectra are the ${ }^{13} \mathrm{C}$ isotopologues that are scaled up by a factor of ten. The shades mark the integral velocity range for each line.

Table 1. Emission area and filling factor.

\begin{tabular}{lrr}
\hline \hline Molecule & $\begin{array}{r}\text { Area } \\
(*) \\
\left(\operatorname{arcmin}^{2}\right)\end{array}$ & $\begin{array}{r}\text { Filling factor } \\
\text { relative to }{ }^{13} \mathrm{CO}\end{array}$ \\
\hline${ }^{13} \mathrm{CO}$ & $\begin{array}{r}1186.1 \\
\mathrm{HCO}^{+}\end{array}$ & 1 \\
$\mathrm{HCN}$ & $230.8(534.7)$ & $0.28(0.45)$ \\
$\mathrm{HNC}$ & $207.4(421.3)$ & $0.21(0.36)$ \\
$\mathrm{N}_{2} \mathrm{H}^{+}$ & $75.6(1304.5)$ & $0.17(0.26)$ \\
\hline
\end{tabular}

Notes. ${ }^{(*)}$ The area and filling factors in brackets are measured with the data that are smoothed and regridded to the same angular resolution of $46^{\prime \prime}$ and pixel size $22^{\prime \prime}$ as the ${ }^{13} \mathrm{CO}$ data.

in the velocity range $10<v_{\mathrm{LSR}}<36 \mathrm{~km} \mathrm{~s}^{-1}$. We then convolved and regridded all dense gas maps to the same resolution as the ${ }^{13} \mathrm{CO}$ one. We divided the $\mathrm{HCN}$ integrated intensity map by the
$\mathrm{HCO}^{+}, \mathrm{HNC}, \mathrm{N}_{2} \mathrm{H}^{+}$, and ${ }^{13} \mathrm{CO}$ map to obtain the line ratio maps shown in Fig. 3.

To study the dense gas properties in different evolutionary stages, we divided the filament into four regions based on the molecular line and dust emission maps. The border between Regions A and B is defined by where the narrow filament connects to the extended photon-dominated regions (PDRs, the narrowest part in $\mathrm{HNC}$ and Hi-GAL maps). Regions $\mathrm{B}$ and $\mathrm{C}$ are separated by the $3 \sigma$ closed contours in the $\mathrm{HCO}^{+}$and $\mathrm{HCN}$ maps. Regions $\mathrm{C}$ and $\mathrm{D}$ are separated by the narrowest part between the two strongest peaks in this region in the HNC map. All polygons are further extended to include all emission above $3 \sigma$.

As shown in Figs. 2 and 3, the four sub-regions are: Region A, infrared dark filament dominated; Region B, infrared bright H II regions with PDRs (Urquhart et al. 2011; Anderson et al. 2014); Region C, infrared dark filament dominated; Region D, high-mass star-forming region with a hypercompact 
Table 2. Observed tracers and their corresponding critical and effective densities.

\begin{tabular}{lccc}
\hline \hline Tracer & $\begin{array}{c}E_{\mathrm{u}} / k^{(a)} \\
(\mathrm{K})\end{array}$ & $\begin{array}{c}n_{\text {crit }}{ }^{(b)} \\
\left(\mathrm{cm}^{-3}\right)\end{array}$ & $\begin{array}{c}n_{\text {eff }}{ }^{(c)} \\
\left(\mathrm{cm}^{-3}\right)\end{array}$ \\
\hline${ }^{13} \mathrm{CO}(1-0)$ & 5.3 & 720. & - \\
$\mathrm{HCO}^{+}(1-0)$ & 4.3 & $6.8 \times 10^{4}$ & $9.5 \times 10^{2}$ \\
$\mathrm{H}^{13} \mathrm{CO}^{+}(1-0)$ & 4.2 & $6.2 \times 10^{4}$ & $3.9 \times 10^{4}$ \\
$\mathrm{HCN}^{2}(1-0)$ & 4.3 & $4.7 \times 10^{5}$ & $8.4 \times 10^{3}$ \\
$\mathrm{HNC}(1-0)$ & 4.4 & $1.4 \times 10^{5}$ & $3.7 \times 10^{3}$ \\
$\mathrm{HN}^{13} \mathrm{C}(1-0)$ & 4.2 & $9.6 \times 10^{4}$ & - \\
$\mathrm{N}_{2} \mathrm{H}^{+}(1-0)$ & 4.5 & $6.1 \times 10^{4}$ & $1.0 \times 10^{4}$ \\
\hline
\end{tabular}

Notes. ${ }^{(a)} E_{u} / k$ are from the Cologne Database for Molecular Spectroscopy (CDMS, Müller et al. 2001, 2005; Endres et al. 2016). ${ }^{(b)} n_{\text {crit }}$ are from Shirley (2015) assuming $T_{\mathrm{k}}=10 \mathrm{~K}$, except for ${ }^{13} \mathrm{CO}(1-0)$ and $\mathrm{HN}^{13} \mathrm{C}(1-0)$, which were calculated following the method described by Shirley (2015). We also assume $\mathrm{HN}^{13} \mathrm{C}(1-0)$ shares the same collision rate coefficients as HNC. The relevant data used for the calculation are from Yang et al. (2010) and Dumouchel et al. (2010), and the Python script can be found at https://github.com/ZhiyuZhang/ critical_densities. ${ }^{(c)} n_{\text {eff }}$ assuming $T_{\mathrm{k}}=10 \mathrm{~K}$ are from Shirley (2015).

(HC) H II region, methanol maser, and water maser (Pandian \& Goldsmith 2007; Pandian et al. 2009; Sánchez-Monge et al. 2011; Urquhart et al. 2011; Anderson et al. 2014). Therefore, we propose an evolutionary sequence among these four sub-regions: that Region A and Region C are the youngest, Region D is more evolved, and Region B with PDRs is the most evolved region. The mass of the four sub-regions derived from the Hi-GAL column density map (Fig. 4) are 1.6, 2.6, 6.1, and $4.4 \times 10^{3} M_{\odot}$, respectively. Considering that there are only a few dense cores in Regions $\mathrm{A}$ and $\mathrm{C}$, with such a large mass reservoir it is likely that Regions $\mathrm{A}$ and $\mathrm{C}$ will form massive stars. Regions $\mathrm{A}$ and $\mathrm{C}$ were also classified as massive star-forming regions by a previous study (Dunham et al. 2011).

As shown in Fig. 3 where we overlaid the HCN integrated intensity contours onto line ratio maps and a Hi-GAL dust temperature map, compared to $\mathrm{HCO}^{+}$and $\mathrm{HNC}$, the relative intensity of $\mathrm{HCN}$ increases dramatically in regions with a high temperature (i.e., Regions B and D). For instance, the $\mathrm{HCN} / \mathrm{HNC}$ ratio remains relatively constant $(\lesssim 2)$ in Regions A and $\mathrm{C}$, where the dust temperature is $T_{\text {dust }} \lesssim 18 \mathrm{~K}$, while in Regions B and D the dust temperature rises to $T_{\text {dust }} \gtrsim 20 \mathrm{~K}$ and the $\mathrm{HCN} / \mathrm{HNC}$ ratio also increases to $\gtrsim 3$. Similar trends can also be seen in the $\mathrm{HCN} / \mathrm{HCO}^{+}$ratio map, where the $\mathrm{HCN} / \mathrm{HCO}^{+}$ ratio is approximately a factor of three higher in Region $\mathrm{B}$ than in Region C.

\subsection{Column density}

Following the standard method described in other works (e.g., Eq. (15.37) in Wilson et al. 2013, appendix in Caselli et al. 2002 and Feng et al. 2016), we estimated the column density of different molecules in this section.

GMF54, like all GMFs and giant molecular clouds (GMCs), is an active site of star formation with warm, IR-bright areas with embedded sources, and cool, IR-dark areas (Sect. 3.1). This obviously implies a complex temperature structure that has an influence on the determination of the column density. In addition, abundance variations can arise from chemistry and from the radiation field. If the local thermal equilibrium (LTE) assumption is valid and the density is larger than the critical or effective density of the transition, the gas kinetic temperature $\left(T_{\text {kin }}\right)$ corresponds to the excitation temperature $\left(T_{\mathrm{ex}}\right)$ for the molecular lines (which is assumed to be equal for all species). If gas and dust are well mixed, this temperature should also correspond to the dust temperature $\left(T_{\text {dust }}\right)$. However, LTE conditions are not present and the density varies from low values in the diffuse gas phase, mainly seen in ${ }^{12} \mathrm{CO}$, to high values in dense clumps in the molecular clouds, traced by dust and the lines of $\mathrm{HCO}^{+}, \mathrm{HCN}, \mathrm{HNC}$ (and their isotopologues), and $\mathrm{N}_{2} \mathrm{H}^{+}$. The temperature also varies from a few kelvins inside cold, dense clumps to a few tens of kelvins in UV-heated PDRs. In the following, we determine the excitation temperature and optical depth in different ways, using our observational data sets or literature values.

Assuming that the ${ }^{12} \mathrm{CO}(1-0)$ is optically thick, Zhang et al. (2019) estimated the excitation temperature $T_{\mathrm{ex}}$ for $13 \mathrm{GMFs}$ from $T_{\mathrm{mb}}\left({ }^{12} \mathrm{CO}\right)$, and they obtained the mean value of $\sim 10 \mathrm{~K}$ with a standard deviation of $2.5 \mathrm{~K}$. Since we do not have ${ }^{12} \mathrm{CO}(1-0)$ observation with compatible angular resolution with GRS for GMF54, we assume ${ }^{13} \mathrm{CO}$ emission to be optically thin and adopt the mean excitation temperature $T_{\mathrm{ex}}=10 \mathrm{~K}$ from Zhang et al. (2019). We discuss the uncertainty introduced by the possible optical depth of ${ }^{13} \mathrm{CO}(1-0)$ in Sect. 4.5.

For $\mathrm{HCN}(1-0)$ and $\mathrm{N}_{2} \mathrm{H}^{+}(1-0)$, we applied a hyperfine structure (HFS) fit pixel-by-pixel using PySpecKit (Ginsburg \& Mirocha 2011) to derive the optical depth $\tau$ and the excitation temperature $T_{\text {ex }}$. When fitting the HFS, we set a lower boundary for $T_{\text {ex }}$ as $4 \mathrm{~K}$. As the HFS fit only produce $\tau \times T_{\text {ex }}$, a lower boundary smaller than $4 \mathrm{~K}$ for $T_{\mathrm{ex}}$ does not change the results in high signal to noise $(\mathrm{S} / \mathrm{N})$ ratio regions but produces large $\tau(>1)$ in low S/N regions (along the $3 \sigma$ contour in Fig. 2), which is not physical. Since our maps are over sampled $\left(32^{\prime \prime} F W H M\right.$ beam and $8^{\prime \prime}$ pixel size), we further convolve the $T_{\text {ex }}$ map and the $\tau$ map into a Gaussian FWHM beam of 32" (Gaussian kernel $~ 13.59^{\prime \prime}$ ). The derived final $T_{\mathrm{ex}}$ and $\tau$ maps including a few selected spectra to show the fit results for $\mathrm{HCN}(1-0)$ and $\mathrm{N}_{2} \mathrm{H}^{+}(1-0)$ are shown in Figs. A.1 and A.2. The excitation temperatures of $\mathrm{HCN}(1-0)$ are between 4 and $74 \mathrm{~K}$ with the highest value at the PDR in Region B, as we expected. The optical depths of $\mathrm{HCN}(1-0)$ are between 0.1 and 3.5 with the highest value in Region $\mathrm{C}$ and $\mathrm{D}$. For $\mathrm{N}_{2} \mathrm{H}^{+}(1-0)$, the derived $T_{\mathrm{ex}}$ is between 4 and $29 \mathrm{~K}$ and $\tau$ is between 0.1 and 2.4 .

For $\mathrm{HCO}^{+}(1-0)$ and $\mathrm{HNC}(1-0)$, since we only observed one transition for the whole filament, we cannot determine the excitation temperatures from our observations directly. Therefore, we assume that $\mathrm{HCO}^{+}(1-0)$ and $\mathrm{HNC}(1-0)$ share the same excitation temperature as $\mathrm{HCN}(1-0)$. We discuss the uncertainty of this in Sect. 4.5. Our observations also cover the $\mathrm{H}^{13} \mathrm{CO}^{+}(1-0)$ and $\mathrm{HN}^{13} \mathrm{C}(1-0)$ lines; we assume these lines to be optically thin, and to share a similar excitation condition as their ${ }^{12} \mathrm{C}$ counterpart lines. This allows us to estimate the optical depth for $\mathrm{HCO}^{+}(1-0)$ and $\mathrm{HNC}(1-0)$. To do this, we estimated the ${ }^{12} \mathrm{C}$ to ${ }^{13} \mathrm{C}$ abundance ratio following the relation $\left[{ }^{12} \mathrm{C}\right] /\left[{ }^{13} \mathrm{C}\right]=6.2 D_{\mathrm{GC}}[\mathrm{kpc}]+9.0$ (Giannetti et al. 2014). For a Galactocentric distance of $D_{\mathrm{GC}}=7.3 \mathrm{kpc}$ (Ragan et al. 2014), $\left[{ }^{12} \mathrm{C}\right] /\left[{ }^{13} \mathrm{C}\right] \sim 54$. For regions where the ${ }^{13} \mathrm{C}$ line integrated intensity is higher than $5 \sigma$, we calculated $\tau$ for the ${ }^{12} \mathrm{C}$ lines following (Eq. (6) in Giannetti et al. 2014)

$R_{12 / 13}=\frac{\int T_{\mathrm{MB}, 12} \mathrm{~d} v}{\int T_{\mathrm{MB}, 13} \mathrm{~d} v}=\frac{1-\mathrm{e}^{-\tau}}{1-\mathrm{e}^{-\tau / \varphi}}$,

where $\varphi$ is the ${ }^{12} \mathrm{C}$ to ${ }^{13} \mathrm{C}$ relative abundance. We assume that the remaining regions where the ${ }^{13} \mathrm{C}$ line emission is below $5 \sigma$ 

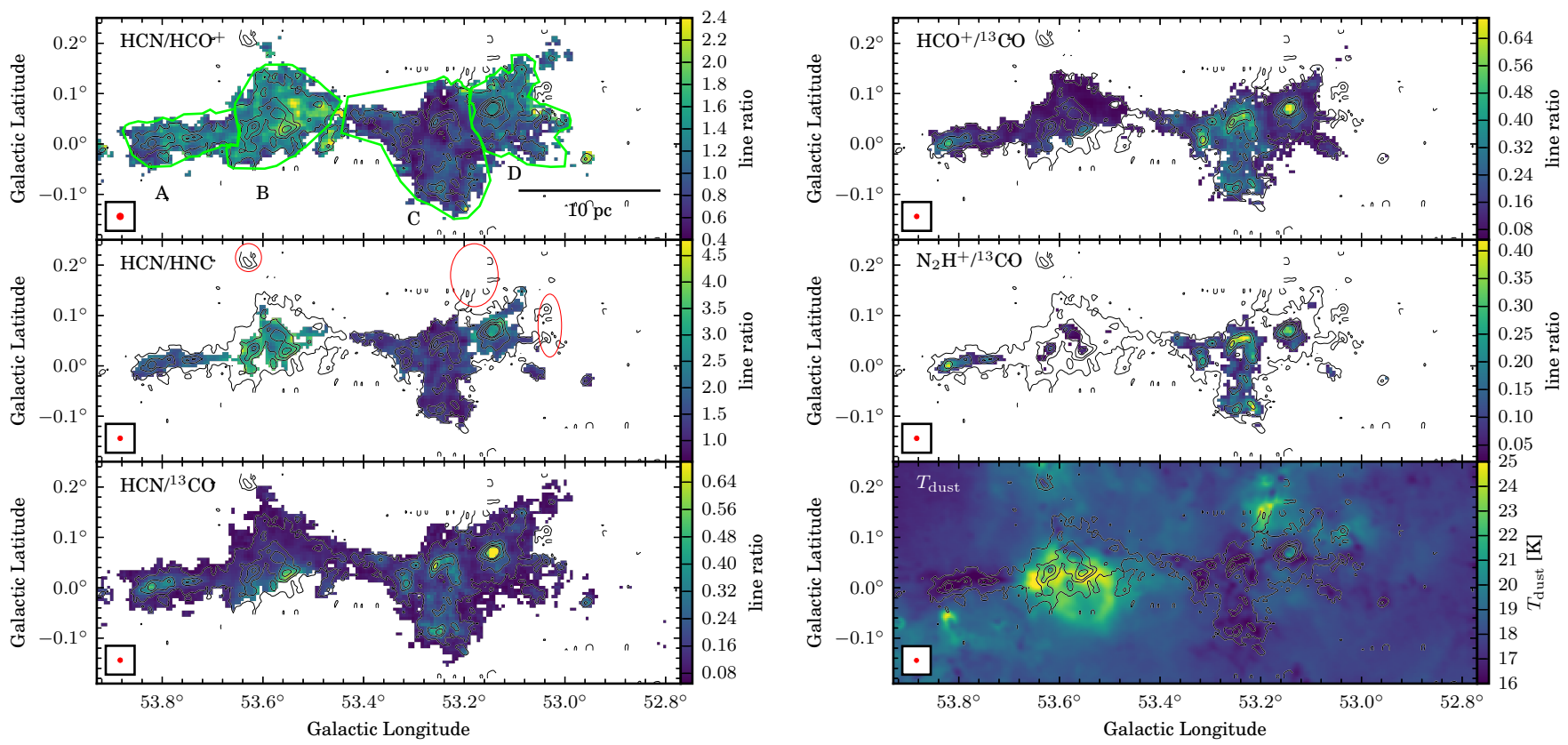

Fig. 3. Spatial distribution of the ratio of the line integrated intensity over $[10,36] \mathrm{km} \mathrm{s}^{-1}$ and the dust temperature (bottom right panel). The contours in all panels trace the $\mathrm{HCN}(1-0)$ integrated intensity, and the contour levels are $3,9,15,25$, and 35 times the noise level $\left(0.4 \mathrm{~K} \mathrm{~km} \mathrm{~s}^{-1}\right)$. The beam of the line ratio maps $\left(46^{\prime \prime}\right)$ is marked in the bottom left of each panel. The polygons and ellipses are the same as in Fig. 2.
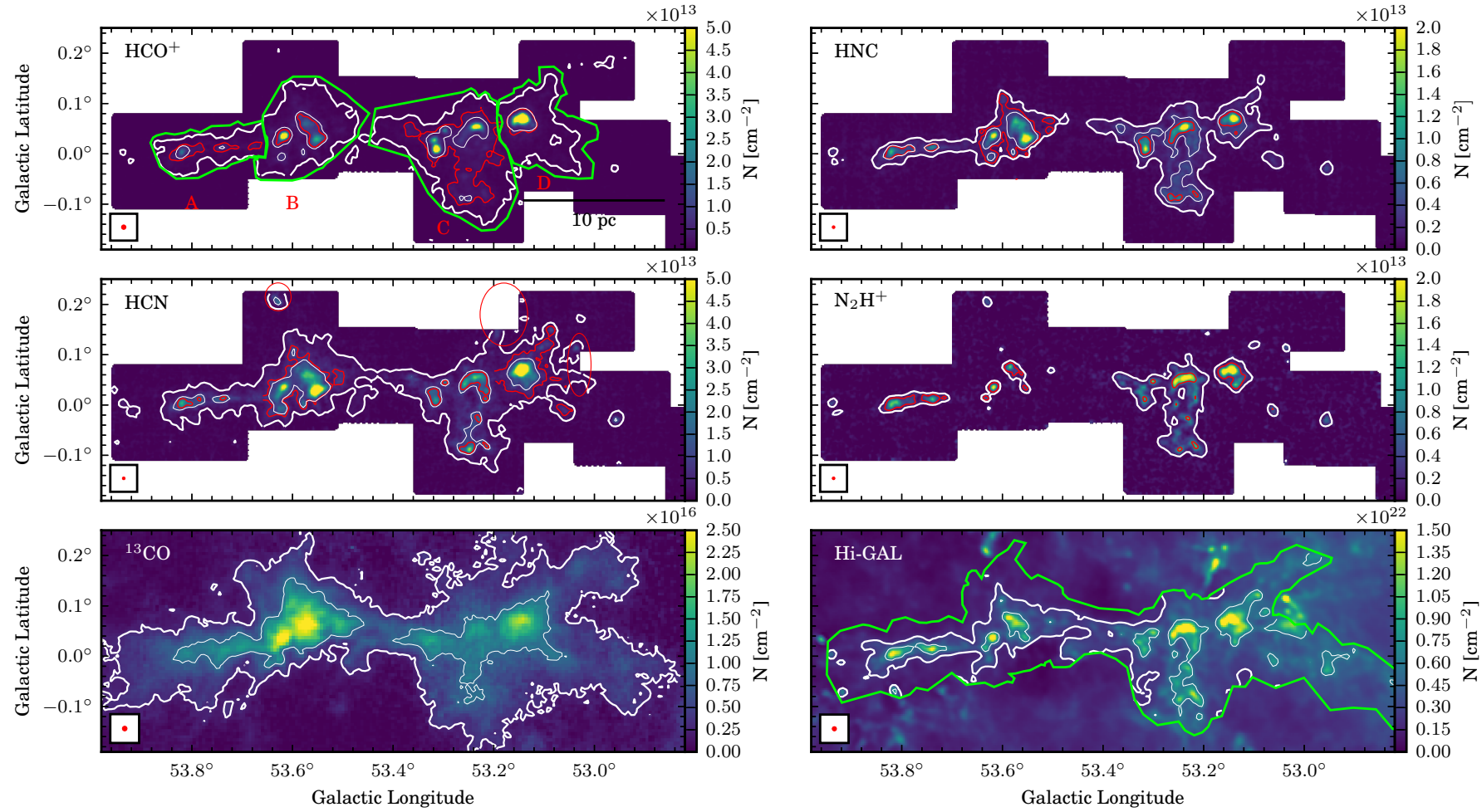

Fig. 4. Column density maps of $\mathrm{HCO}^{+}, \mathrm{HCN},{ }^{13} \mathrm{CO}, \mathrm{HNC}$, and $\mathrm{N}_{2} \mathrm{H}^{+}$, and molecular hydrogen column density map derived from $\mathrm{Hi}-\mathrm{GAL}$ dust continuum emission. The thick white contours in each panel outline the column densities we used to construct the N-PDFs, and the thin white contours mark the optimal column density threshold ( $N_{\min }$ for the optimal power-law fit) for the whole filament. The red contours in the dense gas molecular line panels mark $N_{\min }$ for the power law for the sub-regions (see Sect. 3.3). The green polygon in the Hi-GAL panel outlines the area we used to construct the N-PDFs from the dense gas tracers and continuum emission in the following sections. The beam of the column density maps (43" for Hi-GAL, $46^{\prime \prime}$ for ${ }^{13} \mathrm{CO}$, and $32^{\prime \prime}$ for the other panels) is marked in the bottom left of each panel. The polygons in the $\mathrm{HCO}^{+}$panel and ellipses in the HCN panel are the same as in Fig. 2. 
are optically thin. Since the derived $\tau$ maps are calculated pixel by pixel, due to the limited $\mathrm{S} / \mathrm{N}$ of the data, the $\tau$ value could vary significantly between two neighboring pixels, which is not physically plausible. To avoid this pixelization problem, we further convolved the $\tau$ map into a Gaussian FWHM beam of 32" (Gaussian kernel $\sim 13.59^{\prime \prime}$ ), which is the beam of our $3 \mathrm{~mm}$ data.

We took into account the optical depth and estimated the column densities of $\mathrm{HCO}^{+}, \mathrm{HCN}, \mathrm{HNC}$, and $\mathrm{N}_{2} \mathrm{H}^{+}$with the derived $T_{\text {ex }}$. For $\mathrm{HCO}^{+}$and $\mathrm{HNC}$, limited by the $\mathrm{S} / \mathrm{N}$ of the ${ }^{13} \mathrm{C}$ line emission (Fig. A.3), only a small percentage (8 and $2 \%$, respectively) of the column density maps were corrected for optical depth with the factor $\tau /(1-\exp (-\tau))>1.01$. Since the abundances of these molecules have large uncertainties (e.g., Gerner et al. 2014), we do not convert the column density of the molecules to molecular hydrogen. Instead, we discuss directly the column density distribution of different molecules, and the physical properties of molecular hydrogen traced by these tracers. The column density distributions of these tracers represents the distribution of the molecular cloud at the densities traced by these tracers.

\subsection{Column density probability density function}

We regridded all the column density maps of the dense gas tracers (Fig. 4) to the same angular resolution as the ${ }^{13} \mathrm{CO}$ map (FWHM beam size of $46^{\prime \prime}$ and pixel size of $22^{\prime \prime}$ ) and constructed the column density probability density functions (N-PDFs) of the filament. Figure 5 presents the N-PDFs for the entire filament traced by different molecular lines. All column density values lower than $5 \sigma$ ( $N$ Threshold in Table 3 corresponding to the thick closed contours in Fig. 4) were ignored when calculating the N-PDFs. We also normalized all N-PDFs by the mean column density of each tracer ( $\langle N\rangle$ in Table 3$)$. We also constructed the N-PDFs of the tracers without the optical depth correction shown in Fig. B.1.

As shown in Fig. 5, qualitatively the ${ }^{13} \mathrm{CO}$ N-PDF resembles a log-normal shape, while the N-PDFs of $\mathrm{HCO}^{+}, \mathrm{HCN}, \mathrm{HNC}$, and $\mathrm{N}_{2} \mathrm{H}^{+}$are closer to a broken power-law shape. We fit the $\mathrm{N}$-PDFs with different functions to quantify their shapes. We fit two power laws $\left(p(x) \propto x^{-s}\right)$ to all N-PDFs. We first fit the NPDFs with an optimal power law to the high column density tail from an optimal column density threshold. The fitting procedure creates power-law fits starting from each unique value in the data, and the optimal column density $N_{\min }$ is the one that results in the minimal Kolmogorov-Smirnov (KS) distance between the data and the fit. The fitting was performed with the python package Powerlaw $^{8}$ (Alstott et al. 2014). The obtained $N_{\text {min }}$ (marked with the thin white contours in Fig. 4) and power-law index $s$ are listed in Table 3. Among different tracers, the N-PDF of $\mathrm{HCO}^{+}$has the lowest power-law index of 2.18 , and $\mathrm{N}_{2} \mathrm{H}^{+}$has the highest index of 4.15. We further fit a power-law function that includes all data points to each N-PDF, and all the derived power-law indices are quite similar to each other (between 1.95 and 2.15), which are lower than the ones using the optimal column density threshold $N_{\text {min }}$.

We also fit a single log-normal function ${ }^{9}$ to ${ }^{13} \mathrm{CO}$ N-PDF and derived a width of 0.51 . The errors of the log-normal fitting

\footnotetext{
8 https://github.com/jeffalstott/powerlaw

9 The lognorm function in the Python package SciPy was used to perform the fit (https://docs.scipy.org/doc/scipy/reference/ generated/scipy.stats. lognorm.html).
}

are estimated with a bootstrap method in which we ran the fitting procedure 100 times while removing $10 \%$ of the data points randomly in each run, and we took the difference between the maximum and minimum width as the error of the fit. We cannot identify a clear peak in N-PDF, thus the width we derived could have large uncertainties. A simple model comparison performed with the python package Powerlaw shows the ${ }^{13} \mathrm{CO}$ N-PDF can be better described by a log-normal function than a power law with a loglikelihood ratio of 22 (log-normal over power law) and $p$-value $<0.001$.

To study the dense gas properties in different evolutionary stages, we constructed the dense gas N-PDFs for each sub-region (Fig. 2). Since we only compare between the dense gas tracers, the N-PDFs were constructed with the column density maps at the original resolution ( 8 " pixel size and $32^{\prime \prime}$ beam size). The derived N-PDFs of the dense gas tracers for each sub-region are shown in Fig. 6.

Qualitatively, the N-PDFs of each dense gas tracer show clear differences from region to region. As we proposed in Sect. 3.1, Region A and Region C are at an earlier relative quiescent stage, Region D is more evolved with a HCH II region, and Region B with PDRs is the most evolved region. An evolutionary trend can be seen in some molecular lines, for instance, the HCN and HNC N-PDFs resemble more of a break power-law shape in the younger regions (Regions $\mathrm{C}$ and $\mathrm{A}$ ) and an almost straight single power-law shape in the more evolved Region D. To quantify this evolutionary trend, we fit an optimal powerlaw function to each N-PDF with the python package Powerlaw (Alstott et al. 2014). The obtained optimal column density $N_{\text {min }}$ for each sub-region (marked with the thin red contours in Fig. 4) and power-law indices $s$ are listed in Table 3. As shown in the top panel of Fig. 7, from quiescent regions to $\mathrm{HCH}$ II regions, the power-law slope becomes flatter systematically, then from $\mathrm{HCH}$ II regions to more evolved PDRs the power-law slope steepens again. The lowest contour in the $\mathrm{HCO}^{+}, \mathrm{HCN}$, and $\mathrm{HNC}$ column density maps for these sub-regions are not closed. However, when we fit the N-PDFs with the optimal power law, the optimal column density thresholds $\left(N_{\min }\right)$, as marked with red contours in Fig. 4, are closed contours except in $\mathrm{HCO}^{+}$for Region $\mathrm{C}$ and $\mathrm{HCN}$ for Region D. If we fit the power law from the close contour value $\left(\mathrm{HCO}^{+} 2.2 \times 10^{12} \mathrm{~cm}^{-2}\right.$, HCN $3.71 \times 10^{12} \mathrm{~cm}^{-2}$ ) for these two region, the index for $\mathrm{HCO}+$ (Region C) increases by 0.03 and decreases by 0.01 for $\mathrm{HCN}$ (Region D).

To investigate the evolution of the N-PDFs, we estimated the virial parameter $\alpha_{\text {virial }}$ of each sub-region following the method described in Bertoldi \& McKee (1992). To eliminate the uncertainty brought in by the abundances of the dense gas tracers, we estimated the mass of each sub-region with the Hi-GAL column density map (Fig. 4). For $\mathrm{HCO}^{+}$and $\mathrm{HNC}$, we fit the spectra with a Gaussian profile to derive the velocity dispersion. For $\mathrm{HCN}$ and $\mathrm{N}_{2} \mathrm{H}^{+}$we fit the HFS to derive the velocity dispersion. All fittings were performed with PySpecKit (Ginsburg \& Mirocha 2011). Assuming a circular shape, we estimated the effective radius from the projected pixel area of each sub-region. As shown in Fig. 7 where the power-law indices are plotted as a function of the virial parameters, the virial parameters of all regions and tracers are around or smaller than two.

There is no clear correlation between the virial parameters and the power-law slope (Fig. 7). Among all four molecular lines, $\mathrm{N}_{2} \mathrm{H}^{+}$always has the lowest virial parameter, which makes sense, since $\mathrm{N}_{2} \mathrm{H}^{+}$traces the dense cores that are gravitationally bound. 
Table 3. Summary of the fitting results of the N-PDFs.

\begin{tabular}{|c|c|c|c|c|c|}
\hline Tracers & $\begin{array}{l}N \text { Threshold } \\
\times 10^{12} \mathrm{~cm}^{-2} \\
\end{array}$ & $\begin{array}{c}\langle N\rangle^{(b)} \\
\times 10^{12} \mathrm{~cm}^{-2} \\
\end{array}$ & $\begin{array}{l}\text { Optimal power-law } \\
\text { index } s \\
\end{array}$ & $\begin{array}{l}\text { Single power-law } \\
\text { index } s^{*}\end{array}$ & $\begin{array}{r}N_{\min }{ }^{(c)} \\
\times 10^{12} \mathrm{~cm}^{-2} \\
\end{array}$ \\
\hline \multicolumn{6}{|c|}{ The whole filament } \\
\hline${ }^{13} \mathrm{CO}$ & 2700 & 6500 & $3.97 \pm 0.08$ & $2.36 \pm 0.02$ & 8694 \\
\hline $\mathrm{HCO}^{+}$ & 0.65 & 3.2 & $2.18 \pm 0.07$ & $2.00 \pm 0.02$ & 4.4 \\
\hline $\mathrm{HCN}$ & 1.5 & 6.5 & $2.72 \pm 0.08$ & $1.95 \pm 0.03$ & 8.4 \\
\hline $\mathrm{HNC}$ & 0.93 & 2.9 & $2.92 \pm 0.09$ & $2.15 \pm 0.03$ & 2.9 \\
\hline $\mathrm{N}_{2} \mathrm{H}^{+}$ & 1.4 & 4.8 & $4.15 \pm 0.41$ & $2.00 \pm 0.04$ & 10.3 \\
\hline \multicolumn{6}{|c|}{ Region A } \\
\hline $\mathrm{HCO}^{+}$ & 1.3 & 3.0 & $3.12 \pm 0.10$ & - & 2.3 \\
\hline $\mathrm{HCN}$ & 3.2 & 8.4 & $5.15 \pm 0.39$ & - & 1.4 \\
\hline HNC & 1.6 & 3.5 & $3.54 \pm 0.16$ & - & 3.4 \\
\hline $\mathrm{N}_{2} \mathrm{H}^{+}$ & 2.8 & 6.2 & $2.52 \pm 0.09$ & - & 2.8 \\
\hline \multicolumn{6}{|c|}{ Region B } \\
\hline $\mathrm{HCO}^{+}$ & 1.3 & 4.8 & $2.61 \pm 0.08$ & - & 7.0 \\
\hline $\mathrm{HCN}$ & 3.2 & 11.0 & $2.42 \pm 0.03$ & - & 6.2 \\
\hline $\mathrm{HNC}$ & 1.6 & 4.8 & $2.19 \pm 0.03$ & - & 1.6 \\
\hline $\mathrm{N}_{2} \mathrm{H}^{+}$ & 2.8 & 6.2 & $2.62 \pm 0.12$ & - & 2.8 \\
\hline \multicolumn{6}{|c|}{ Region C } \\
\hline $\mathrm{HCO}^{+}$ & 1.3 & 4.3 & $2.68 \pm 0.03$ & - & 2.1 \\
\hline $\mathrm{HCN}$ & 3.2 & 8.0 & $3.76 \pm 0.12$ & - & 1.2 \\
\hline $\mathrm{HNC}$ & 1.6 & 3.8 & $3.24 \pm 0.11$ & - & 5.6 \\
\hline $\mathrm{N}_{2} \mathrm{H}^{+}$ & 2.8 & 6.6 & $3.89 \pm 0.17$ & - & 9.4 \\
\hline \multicolumn{6}{|c|}{ Region D } \\
\hline $\mathrm{HCO}^{+}$ & 1.3 & 8.8 & $1.76 \pm 0.04$ & - & 3.4 \\
\hline $\mathrm{HCN}$ & 3.2 & 1.5 & $2.05 \pm 0.03$ & - & 3.2 \\
\hline HNC & 1.6 & 4.6 & $2.41 \pm 0.10$ & - & 4.4 \\
\hline $\mathrm{N}_{2} \mathrm{H}^{+}$ & 2.8 & 9.9 & $2.23 \pm 0.07$ & - & 4.4 \\
\hline
\end{tabular}

Notes. ${ }^{(a)}$ Five $\sigma$ threshold for the N-PDFs and the thick white contours in Fig. $4 .{ }^{(b)}$ The mean column density above $5 \sigma$ and used to normalize the column density for the N-PDFs. ${ }^{(c)}$ The optimal column density determined by the program Powerlaw.

Virial parameters of Regions A and C measured with all four tracers are all $\lesssim 1$, indicating the gases traced by these four tracers in these two relative quiescent regions are all at gravitational virial equilibrium and could be undergoing gravitational collapse (e.g., Bertoldi \& McKee 1992; McKee \& Zweibel 1992). For the more evolved Regions B and D, while the gas traced by $\mathrm{N}_{2} \mathrm{H}^{+}$is still gravitationally bound $\left(\alpha_{\text {virial }} \lesssim 1\right)$, the gas traced by $\mathrm{HCO}^{+}$shows much larger $\alpha_{\text {virial }}$ and could be confined by pressure (Bertoldi \& McKee 1992).

\section{Discussion}

We discuss the N-PDF and line ratio results in this section. The uncertainties of our results are also discussed.

\subsection{N-PDFs of the whole filament}

As mentioned in Sect. 1, there is only one prior dense gas N-PDF study. Schneider et al. (2016) studied the N-PDFs of Cygnus X with Herschel dust continuum, ${ }^{12} \mathrm{CO}(1-0),{ }^{13} \mathrm{CO}(1-0), \mathrm{C}^{18} \mathrm{O}(1-$ 0 ), and the dense gas tracers $\mathrm{N}_{2} \mathrm{H}^{+}(1-0)$ and $\mathrm{CS}(2-1)$. They found that compared to the dust N-PDF, the N-PDFs derived from $\mathrm{CO}$ observations can recover a log-normal shape in the lower column density regime, whereas the N-PDFs derived from dense gas tracers only recover a power-law tail in the high column density regime. Although the absolute column density depends on the abundance and excitation temperature adopted, the power-law index of the dense gas is robust $\left(-1.41\right.$ for $\mathrm{N}_{2} \mathrm{H}^{+}$, and -1.56 for CS). Compared to Schneider et al. (2016), our observation is deeper; for instance, the $3 \sigma$ level of the $\mathrm{N}_{2} \mathrm{H}^{+}(1-$ 0 ) observation from Schneider et al. (2016) corresponds to a $N\left(\mathrm{H}_{2}\right)$ of $\sim 1 \times 10^{22} \mathrm{~cm}^{-2}$, while if we apply the same abundance, the $3 \sigma$ level of our $\mathrm{N}_{2} \mathrm{H}^{+}(1-0)$ observation corresponds to an $N\left(\mathrm{H}_{2}\right)$ of $3.6 \times 10^{21} \mathrm{~cm}^{-2}$. The ${ }^{13} \mathrm{CO} \mathrm{N-PDF}$ can be better described with a log-normal function, but we still could not recover the log-normal peak of the N-PDFs.

Wang et al. (2020) studied the N-PDFs of the atomic and molecular gas in the giant molecular filament GMF38a, and found that the N-PDF of H I emission has the smallest width, followed by the cold neutral media traced by $\mathrm{H}$ I self absorption (HISA), and the ${ }^{13} \mathrm{CO} \mathrm{N}-\mathrm{PDF}$ has the largest width. It seems that the width of the N-PDF is correlated with the density it traces.

The power-law indices derived by fitting all data points in the N-PDFs are quite similar for all molecular lines, between 1.95 and 2.15 ( $s^{*}$ in Table 3$)$. On the other hand, the optimal 

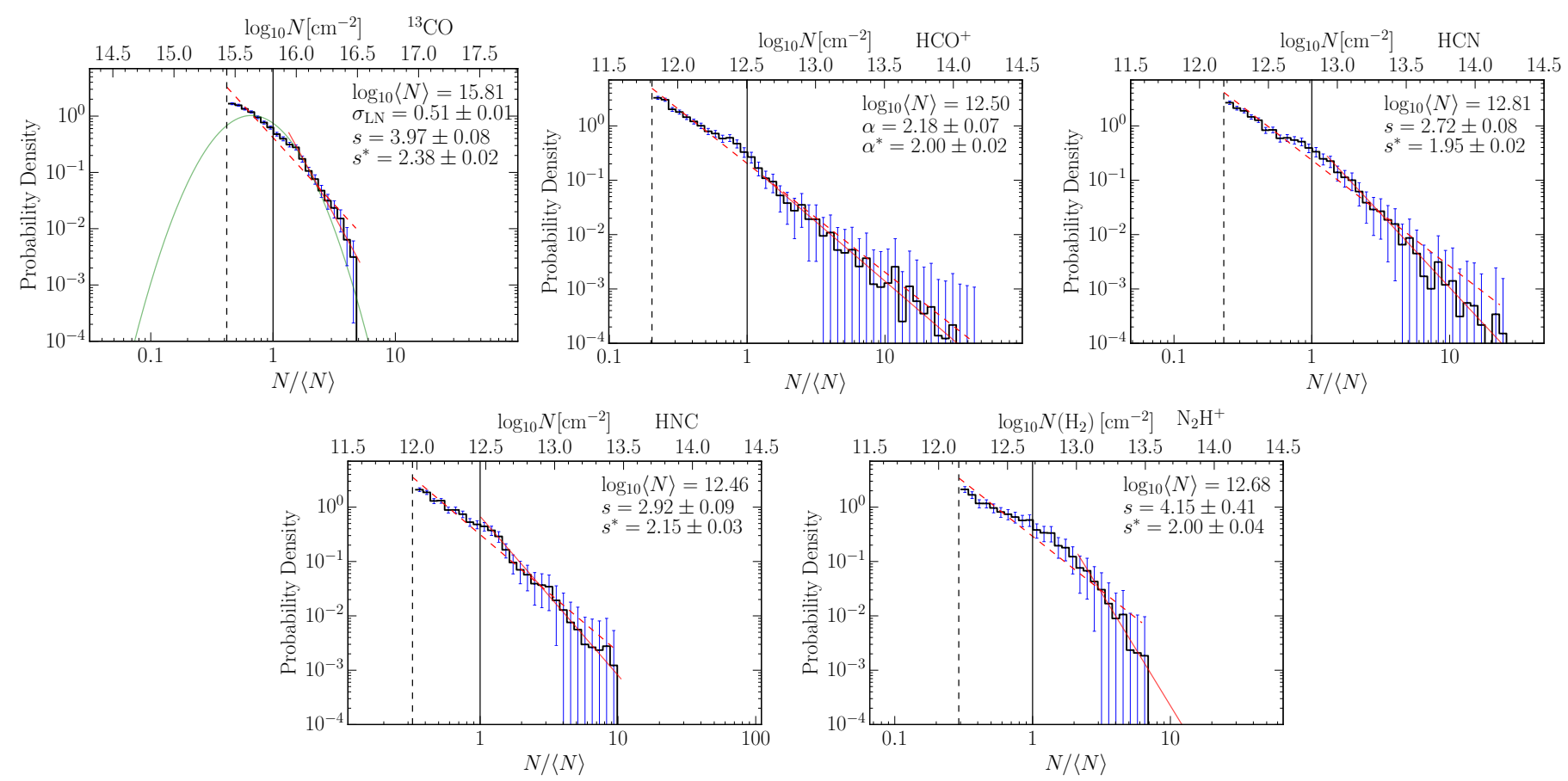

Fig. 5. Probability density functions of the ${ }^{13} \mathrm{CO}, \mathrm{HCO}^{+}, \mathrm{HCN}, \mathrm{HNC}$, and $\mathrm{N}_{2} \mathrm{H}^{+}$column density for the whole filament. The dashed vertical lines in each panel mark the column density threshold ( $5 \sigma$ level), and the solid vertical lines mark the mean column densities. We list in each panel the mean column density $\langle N\rangle$, the power-law index $s$ (the optimized fit, solid line), and $s^{*}$ (the fit to all data, dashed line). For the ${ }^{13} \mathrm{CO} N-\mathrm{PDF}$, we also list the log-normal width $\sigma_{\mathrm{LN}}$. Error bars are calculated from Poisson statistics.

power-law indices ( $s$ in Table 3) are different between different lines. A power-law tail in the N-PDF requires a power-law distribution in the density structure. Though such a density configuration can be achieved by a hydrostatic configuration, where the power law arises from a balance of gravitational forces and pressure gradients, it is more likely that in a massive GMF self-gravity is the dominant process. Large-scale collapse of filaments and clumps as well as small-scale collapse of high-density cores then gives rise to the power-law slopes, seen in the different tracers. Following the method described in Appendix D of Schneider et al. (2016), we correlate the slope of the power-law tail $s$ and the exponent $\alpha$ of a spherical (for clumps and cores, $\rho(r) \propto r^{-\alpha_{\mathrm{c}}}$ ) and a cylindrical (for filaments, $\rho(r) \propto r^{-\alpha_{\mathrm{f}}}$ ) density distribution via $\alpha_{c}=1+2 / s$ and $\alpha_{f}=1+1 / s$, respectively (see also Appendx C). Similar calculations are also discussed by Federrath \& Klessen (2013), Fischera (2014), and Myers (2015). The power-law indices $s^{*}$ for the dense gas are all around 2, thus the radial density profile $\alpha_{c}$ is around 2 and $\alpha_{f}$ around 1.5. These values are fully consistent with the collapse of an isothermal sphere (e.g., Shu 1977) and with a self-gravitating filament model (e.g., Myers 2015).

The N-PDFs of molecular clouds in the nearby galaxy M33 derived from ${ }^{12} \mathrm{CO}$ observations (Druard et al. 2014; Corbelli et al. 2018) peak at a lower column density, but the shape and width is similar to the ${ }^{13} \mathrm{CO}$ one in Fig. 5. Our study provides the foundation to interpret future high angular resolution dense gas tracer observations toward nearby galaxies with the Atacama Large Millimeter/submillimeter Array.

\subsection{N-PDFs and evolutionary stages}

Previous observations show that clouds without star formation have log-normal shape N-PDF with little or no excess in the high column density tail, while active star-forming clouds present prominent power-law-like wings (e.g., Kainulainen et al. 2009; Schneider et al. 2013). Furthermore, the slope index of the power-law tail is correlated with star formation activities, as clumps with flatter power-law tails are more efficient at forming Class 0 protostars (Sadavoy et al. 2014; Stutz \& Kainulainen 2015). Louvet et al. (2014) demonstrated that the star formation rate per free fall time steeply decreases with the virial parameter. The dense gas N-PDFs for the sub-regions further confirm this since more quiescent regions have flatter N-PDFs (Table 3). Although with a large scatter, we also find a correlation between the evolutionary stages of sub-regions and N-PDF power-law indices (Fig. 7). Sources at early quiescent stages have a lower fraction of high density gas, which would result in a steeper power-law slope, while in high-mass star-forming regions with a $\mathrm{HCH}$ II region the fraction of high density gas is higher, which results in a flatter power-law slope. When the source evolves into the PDR stage, the feedback processes from high-mass stars could reduce the dense gas fraction, and the power-law slope of the N-PDF is also steeper again.

\subsection{Comparison to the dust continuum N-PDF}

To compare with the N-PDFs we derived from dense gas tracers, we also constructed the N-PDF from the Herschel dust continuum column density map. We convolved and regridded the dust column density map to the same angular resolution and pixel size as the GRS ${ }^{13} \mathrm{CO}(1-0)$ map, and constructed the N-PDF shown in Fig. 8. We also fitted an optimal power-law function and a lognormal function (with a closed contour $N\left(\mathrm{H}_{2}\right)=6.0 \times 10^{21} \mathrm{~cm}^{-2}$ ) to the dust N-PDF. Since there is no closed contour in the low column density regime of the dust N-PDF, and the log-normal width is not reliable (Alves et al. 2017), we only compare the power-law slope and the column density range to the ones traced by molecular lines. 

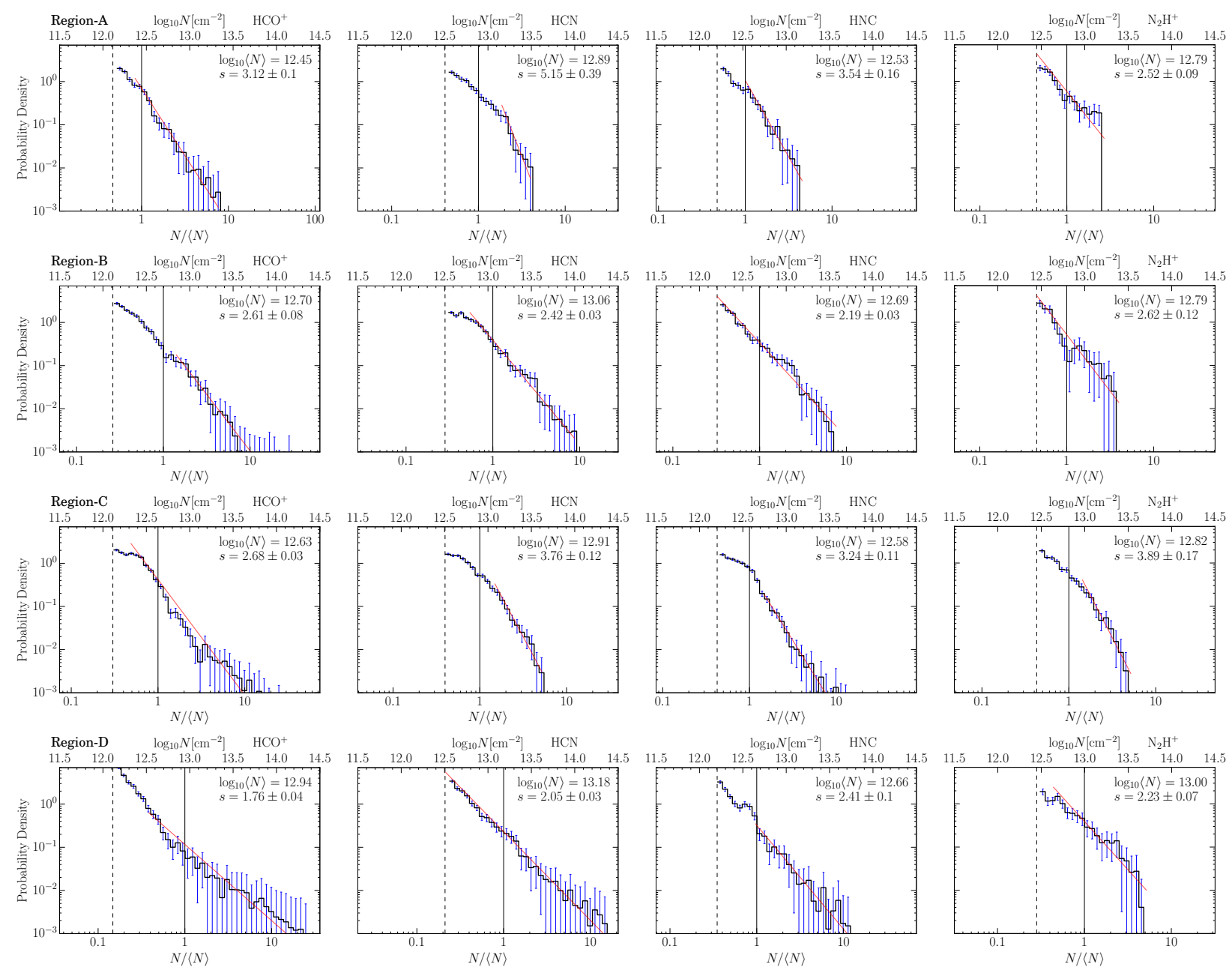

Fig. 6. Probability density functions of the ${ }^{13} \mathrm{CO}, \mathrm{HCO}^{+}, \mathrm{HCN}, \mathrm{HNC}$, and $\mathrm{N}_{2} \mathrm{H}^{+}$column density for Regions A, B, C, and D. The dashed vertical lines in each panel mark the column density threshold, and the solid vertical lines mark the mean column densities. We list in each panel the mean column density $\langle N\rangle$, the power-law index $s$ (the optimal fit, solid line). Error bars are calculated from Poisson statistics.

Compared to N-PDFs from dense gas tracers, the dust NPDF has a similar power-law slope to $\mathrm{N}_{2} \mathrm{H}^{+}$. Indeed, Figs. 2 and 4 also show that the $\mathrm{N}_{2} \mathrm{H}^{+}$emission follows the dust column density very well, and traces the dense cores (see also Kauffmann et al. 2017). Similar to Schneider et al. (2016), we can also shift the $\mathrm{N}_{2} \mathrm{H}^{+} \mathrm{N}-\mathrm{PDF}$ to "calibrate" the $\mathrm{N}_{2} \mathrm{H}^{+}$abundance with the dust N-PDF, and the "calibrated" abundance of $\mathrm{N}_{2} \mathrm{H}^{+}$is $1.6 \times 10^{-9}$. This calibrated abundance is similar to the abundance Gerner et al. (2014) estimated for high-mass protostellar objects.

\subsection{Line ratios}

Integrated intensity ratios of molecular lines are often used to trace molecular gas properties, which can be linked to star formation activities. Numerous surveys have been carried out toward nearby galaxies in the $3 \mathrm{~mm}$ band (Watanabe et al. 2014; Aladro et al. 2015; Meier et al. 2015; Nishimura et al. 2016a,b; JiménezDonaire et al. 2019). We would like to compare our observations of GMF54 with the observed line ratios in such nearby galaxies. Figure 3 shows the line ratio map of GMF54, and we list the averaged line ratios of GMF54 and in the nearby galaxies in Table 4. Most of the observations of dense gas in the nearby galaxies in Table 4 were carried out with single-dish telescopes, where the spatial resolution varies from $\sim 10 \mathrm{pc}$ towards the Large Magellanic Cloud (LMC) to $\sim 24 \mathrm{kpc}$ toward some active galactic nucleus (AGNs). In comparison to this, GMF54 has a size of $68 \mathrm{pc}$ and our observations have a spatial resolution of $0.32 \mathrm{pc}$. Despite the huge spatial resolution differences, the line ratios we derived in GMF54 are in general comparable to those seen in the nearby galaxies.

The HCN/CO ratio is used to trace the dense gas fraction, which is directly related to star formation activity (Gao \& Solomon 2004; Usero et al. 2015; Bigiel et al. 2016). Since we do not have $\mathrm{CO}$ observations, we use the $\mathrm{HCN} /{ }^{13} \mathrm{CO}$ ratio. Compared to nearby galaxies, the $\mathrm{HCN} /{ }^{13} \mathrm{CO}$ ratio in GMF54 is much lower than in nearby galaxies, while Ultraluminous Infrared Galaxies (ULIRGs) present the highest $\mathrm{HCN} /{ }^{13} \mathrm{CO}$ ratio. As discussed by Shirley (2015), Kauffmann et al. (2017), and Pety et al. (2017), HCN traces much lower density than its critical density $n_{\text {crit }}$, and typically traces densities around $\sim 10^{2}-10^{3} \mathrm{~cm}^{-3}$. The HCN flux is also influenced by far ultraviolet (UV) radiation (Pety et al. 2017). Similarly, $\mathrm{HCO}^{+}(1-0)$ also traces only moderate dense gas, and the flux can be affected by far-UV radiation (Shirley 2015; Pety et al. 2017). Thus ULIRGs and AGNs have the highest $\mathrm{HCO}^{+} /{ }^{13} \mathrm{CO}$ ratio, while GMF54 has the lowest ratio.

On the other hand, $\mathrm{N}_{2} \mathrm{H}^{+}$traces the real dense gas fraction with an effective density $n_{\text {eff }} 10^{4} \mathrm{~cm}^{-3}\left(T_{\mathrm{k}}=10 \mathrm{~K}\right)$. The $\mathrm{N}_{2} \mathrm{H}^{+} /{ }^{13} \mathrm{CO}$ ratio, which traces the high density gas, shows similar values in GMF54 and nearby galaxies, except in ULIRGs 
Table 4. Integrated intensity ratios.

\begin{tabular}{|c|c|c|c|c|c|c|c|}
\hline Sources & $\mathrm{HCN} / \mathrm{HCO}^{+}$ & $\mathrm{HCN} / \mathrm{HNC}$ & $\mathrm{HCN} /{ }^{13} \mathrm{CO}$ & $\mathrm{HCO}^{+} /{ }^{13} \mathrm{CO}$ & $\mathrm{N}_{2} \mathrm{H}^{+} /{ }^{13} \mathrm{CO}$ & Telescope & Ref. \\
\hline GMF54 & 1.09 & 1.95 & 0.15 & 0.15 & 0.10 & IRAM $30 \mathrm{~m}$ & 1 \\
\hline W51 arm & 1.29 & 2.78 & 0.35 & 0.27 & 0.06 & IRAM $30 \mathrm{~m}$ & 2 \\
\hline Starbursts & $0.70-1.18$ & $1.93-2.47$ & $0.59-0.84$ & $0.54-1.19$ & $0.06-0.13$ & IRAM $30 \mathrm{~m}$ & 3 \\
\hline AGNs & $0.82-2.02$ & $2.19-3.03$ & $0.81-1.80$ & $0.40-1.24$ & $0.11-0.15$ & IRAM $30 \mathrm{~m}$ & 3 \\
\hline ULIRGs & $1.54-2.39$ & $1.42-3.03$ & $2.29-5.02$ & $0.96-3.27$ & 0.49 & IRAM $30 \mathrm{~m}$ & 3 \\
\hline LMC & $0.40-1.36$ & $1.13-3.36$ & $0.05-0.60$ & $0.13-0.89$ & $0.01-0.11$ & Mopra $22 \mathrm{~m}$ & 4 \\
\hline Dwarf galaxy IC10 & 0.04 & 3.15 & 0.22 & 0.56 & 0.07 & Nobeyama $45 \mathrm{~m}$ & 5 \\
\hline Barred disk & 1.30 & 1.75 & 0.29 & 0.25 & - & IRAM $30 \mathrm{~m}$ & 6 \\
\hline Unbarred disk & 1.29 & 2.52 & 0.25 & 0.27 & - & IRAM $30 \mathrm{~m}$ & 6 \\
\hline
\end{tabular}

References. 1. This work; 2. Watanabe et al. (2014); 3. Aladro et al. (2015); 4. Nishimura et al. (2016b); 5. Nishimura et al. (2016a); 6. EMPIRE project, Jiménez-Donaire et al. (2019).
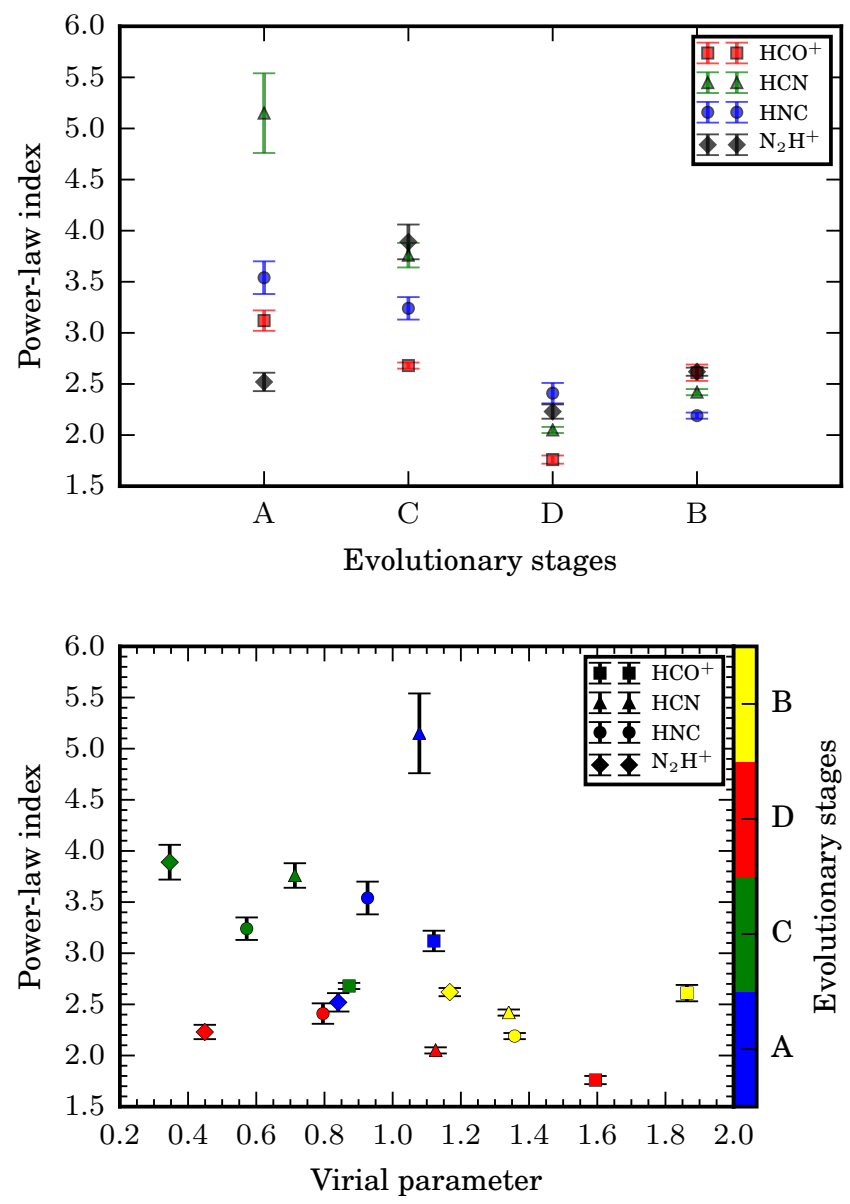

Fig. 7. Power-law indices of the N-PDFs plotted as a function of the evolutionary stages (top panel) and of the virial parameter (bottom panel) the sub-regions. The data points in the bottom panel are colored according to the sub-regions and evolutionary stages: Regions $\mathrm{A}$ and $\mathrm{C}$ are at a relatively early and quiescent stage, Region $\mathrm{D}$ is more evolved with an $\mathrm{HCH}$ II region, and Region B is the most evolved region with PDRs.

which have a much higher $\mathrm{N}_{2} \mathrm{H}^{+} /{ }^{13} \mathrm{CO}$ ratio. Considering ULIRGs are forming many stars, it is reasonable that they have a higher fraction of high density gas. It is surprising that GMF54 has a similar or even higher $\mathrm{N}_{2} \mathrm{H}^{+} /{ }^{13} \mathrm{CO}$ ratio than the starbursts in Table 4. Considering that the filling factor of $\mathrm{N}_{2} \mathrm{H}^{+}$is $\lesssim 10 \%$ (with respect to ${ }^{13} \mathrm{CO}$ ), it is likely that the extragalactic studies are underestimating the true $\mathrm{N}_{2} \mathrm{H}^{+} /{ }^{13} \mathrm{CO}$ ratio.

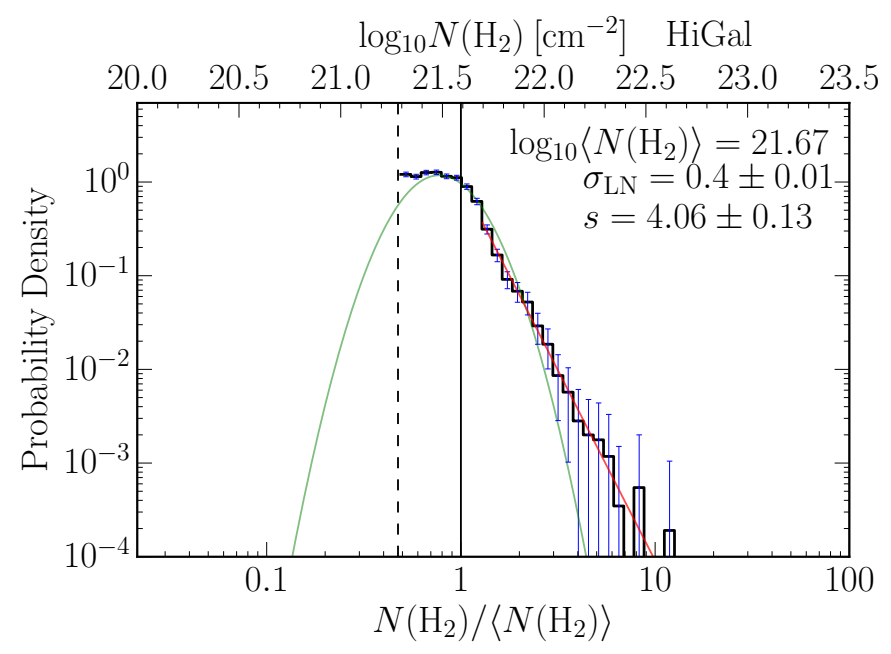

Fig. 8. Probability density functions of the molecular hydrogen column density derived from Hi-GAL (Fig. 2). The dashed vertical line marks the column density threshold, and the solid vertical line marks the mean column density. We list in the figure the mean column density $\left\langle N\left(\mathrm{H}_{2}\right)\right\rangle$, the log-normal (green curve) width $\sigma_{\mathrm{LN}}$, and the power-law index $s$ (solid line). Error bars are calculated from Poisson statistics.

Another useful line ratio is $\mathrm{HCN} / \mathrm{HNC}$, which is proposed as a tracer of evolutionary stages of the molecular cloud because at temperatures $\gtrsim 30 \mathrm{~K} \mathrm{HNC}$ starts to convert to HCN (Schilke et al. 1992; Herbst et al. 2000; Graninger et al. 2014; Hacar et al. 2020). Therefore, the HCN/HNC ratio would increase as temperature increases. As we demonstrate in Fig. 3, the HCN/HNC ratio remains relatively constantly $\lesssim 2$ in infrared dark regions, and increases to $\gtrsim 3$ in the PDRs and around infrared bright $\mathrm{HCH}$ II region. To further demonstrate this, we plotted the HCN/HNC ratio as a function of the dust temperature derived from Hi-GAL (Zucker et al. 2018). A clear correlation between the HCN/HNC ratio and $T_{\text {dust }}$ can be seen in Fig. 9, with a Pearson correlation coefficient of 0.77 and $p$-value $<0.001$. The mean HCN/HNC ratio across the whole GMF54 is also comparable to nearby galaxies.

\subsection{Uncertainties}

Besides the rms noise of the data, the main factors that introduce uncertainties into the column densities and N-PDFs are excitation temperature and optical depth. For $\mathrm{N}_{2} \mathrm{H}^{+}$and $\mathrm{HCN}$ we derive $T_{\mathrm{ex}}$ and $\tau$ from the HFS fitting. The uncertainties of 


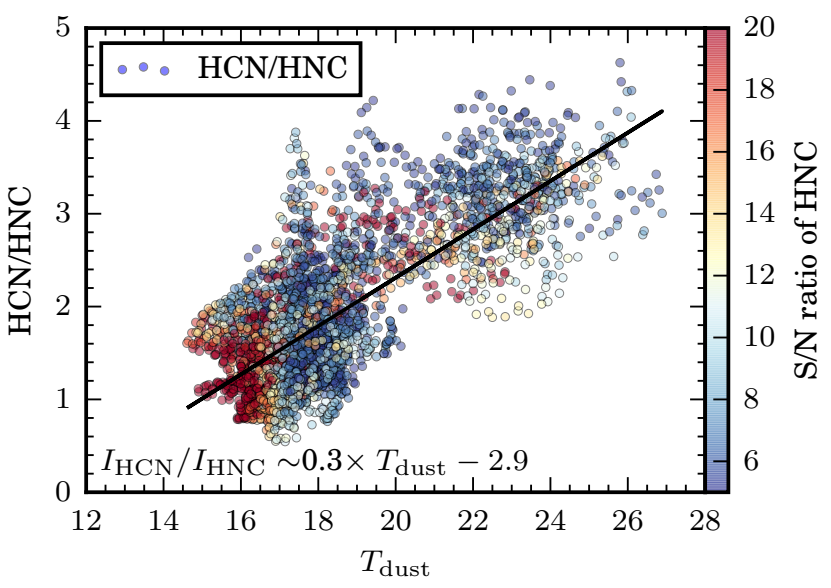

Fig. 9. Integrated intensity ratio of HCN/HNC plotted as a function of the dust temperature for the whole filament pixel by pixel. The points are colored according to the $\mathrm{S} / \mathrm{N}$ of the $\mathrm{HNC}$ integrated intensity. Pixels below $5 \sigma$ were masked out. A linear fit result is plotted and labeled in the figure.

$T_{\text {ex }}$ and $\tau$ of the fitting for $\mathrm{N}_{2} \mathrm{H}^{+}$have a median value of $2 \mathrm{~K}$ and 0.5 , respectively. Therefore, the column density uncertainty of the $\mathrm{N}_{2} \mathrm{H}^{+}$column density brought in by HFS fitting is $\sim 50 \%$. The uncertainties of $T_{\mathrm{ex}}$ and $\tau$ for $\mathrm{HCN}$ have a median value of $1 \mathrm{~K}$ and 0.1 , respectively, which brings in $\sim 10 \%$ uncertainty to the HCN column density estimation.

Since we cannot derive the excitation temperature for $\mathrm{HCO}^{+}$ and HNC directly from our observations, we assume they share the same excitation temperature as HCN. If we assume the excitation temperatures of $\mathrm{HCO}^{+}(1-0)$ and $\mathrm{HNC}(1-0)$ are systematically $50 \%$ higher or lower than $\mathrm{HCN}(1-0)$, the average uncertainty introduced to the column densities of $\mathrm{HCO}^{+}$and $\mathrm{HNC}$ is $\sim 7 \%$. The uncertainties of the N-PDF power-law indices for the whole filament brought in by the excitation temperature are between 0.1 and 0.5 . For the sub-regions, this uncertainty of power-law indices of $\mathrm{HCO}^{+}$is $<0.6$. For $\mathrm{HNC}$, the changes in $\mathrm{T}_{\text {ex }}$ also change the derived $N_{\min }$ for the optimal fit, and further increase the power-law indices for Regions $\mathrm{C}$ and $\mathrm{D}$ by $<0.4$ and Regions $\mathrm{A}$ and $\mathrm{B}$ by $<2$. However, the evolutionary trend of the power-law index shown in Fig. 7 does not change: the quiescent Regions $\mathrm{A}$ and $\mathrm{C}$ show the steepest power-law index, the $\mathrm{HCH}$ II region $\mathrm{D}$ has the flattest power-law index, and Region $\mathrm{B}$ with PDRs has an intermediate power-law index.

To estimate the ${ }^{13} \mathrm{CO}$ column density we adopted a uniform excitation temperature of $10 \mathrm{~K}$, which was derived by Zhang et al. (2019) from estimating the $T_{\mathrm{ex}}$ for $13 \mathrm{GMFs}$ using $T_{\mathrm{mb}}\left({ }^{12} \mathrm{CO}\right)$. They derived a mean excitation temperature of $\sim 10 \mathrm{~K}$ with a standard deviation of $2.5 \mathrm{~K}$, and the ${ }^{13} \mathrm{CO}$ column density uncertainty brought in is $\sim 19 \%$. Another uncertainty source for the ${ }^{13} \mathrm{CO}$ column density is optical depth. Since we do not have a ${ }^{12} \mathrm{CO}$ or $\mathrm{C}^{18} \mathrm{O}$ observation with compatible angular resolution, we cannot estimate the ${ }^{13} \mathrm{CO}(1-0)$ optical depth directly. With a similar angular resolution, Schneider et al. (2016) estimated the ${ }^{13} \mathrm{CO}(1-0)$ optical depth for Cygnus X North and found that the optical depth is negligible and does not influence the N-PDF significantly. Riener et al. (2020) estimated the ${ }^{13} \mathrm{CO}(1-0)$ optical depth for GMF24, and found a median $\tau$ value of $\sim 0.5$. Wang et al. (2020) found that the GRS ${ }^{13} \mathrm{CO}(1-0)$ optical depth for another filament, GMF38a $\left(l \sim 33^{\circ}-37^{\circ}\right)$, is mostly smaller than 1 with a median value of $\sim 0.4$. If we apply this 0.4 to GMF54, the column density increases by $\sim 21 \%$. In total the ${ }^{13} \mathrm{CO}$ column density has an uncertainty of $\sim 40 \%$.

\section{Conclusions}

We studied the dense gas properties of one giant molecular filament, GMF54, with typical dense gas tracers at $3 \mathrm{~mm}$, $\mathrm{HCO}^{+}(1-0), \mathrm{HCN}(1-0), \mathrm{HNC}(1-0)$, and $\mathrm{N}_{2} \mathrm{H}^{+}(1-0)$. The main results are summarized as follows:

1. All dense gas molecular lines trace the extended structure of the filament. The area filling factors with respect to ${ }^{13} \mathrm{CO}(1-$ 0 ) of $\mathrm{HCO}^{+}, \mathrm{HCN}, \mathrm{HNC}$, and $\mathrm{N}_{2} \mathrm{H}^{+}$are, $0.28,0.21,0.17$, and 0.06 , respectively. If we convolve and regrid the dense gas maps to the same angular resolution and pixel size as the ${ }^{13} \mathrm{CO}$ map, the filling factors increase by $\sim 70 \%$.

2. We constructed the N-PDFs of the ${ }^{13} \mathrm{CO}$ column density and the optical depth corrected $\mathrm{HCO}^{+}, \mathrm{HCN}, \mathrm{HNC}$, and $\mathrm{N}_{2} \mathrm{H}^{+}$column densities. While the ${ }^{13} \mathrm{CO}$ N-PDF can be better fitted with a log-normal function, the others are closer to a power-law shape. We fitted power-law functions to the NPDFs. The $\mathrm{HCO}^{+} \mathrm{N}-\mathrm{PDF}$ has the flattest power-law slope. The radial density profile of the filament is estimated to be $\sim 1.5$, which is consistent with the self-gravitating filament model.

3. We studied the N-PDFs for sub-regions of GMF54, and found an evolutionary trend in the N-PDFs, in the sense that more evolved regions have a flatter power-law index.

4. The integrated intensity ratios of the observed molecular lines in GMF54 are comparable to those in nearby galaxies. The $\mathrm{N}_{2} \mathrm{H}^{+} /{ }^{13} \mathrm{CO}$ ratio, which traces the dense gas fraction, has similar values in GMF54 and all nearby galaxies except ULIRGs. A clear correlation between the HCN/HNC ratio and the dust temperature was detected with a Pearson correlation coefficient of 0.74 and $p$-value $<0.001$.

As the largest coherent cold gas structures in our Milky Way, GMFs are excellent candidates to connect studies of star formation on Galactic and extragalactic scales. A sample study with statistic results will help us better understand the physical nature of GMFs.

Acknowledgements. Y.W., H.B., S.B., and J.D.S. acknowledge support from the European Research Council under the Horizon 2020 Framework Program via the ERC Consolidator Grant CSF-648505. H.B. also acknowledges support from the Deutsche Forschungsgemeinschaft in the Collaborative Research Center (SFB 881) The Milky Way System (subproject B1). F.B. acknowledges funding from the European Research Council (ERC) under the European Unions Horizon 2020 research and innovation programme (grant agreement No. 726384/EMPIRE). N.S. acknowledges support from the ANR/France and DFG/Germany project "GENESIS" (ANR-16-CE92-0035-01/DFG1591/2-1) and from the DFG project SFB 956.C.B. gratefully acknowledges support from the National Science Foundation under Award No. 1816715. This research made use of Astropy and affiliated packages, a community-developed core Python package for Astronomy (Astropy Collaboration 2018), Python package SciPy (https://www.scipy. org), APLpy, an open-source plotting package for Python (Robitaille \& Bressert 2012).

\section{References}

Abreu-Vicente, J., Kainulainen, J., Stutz, A., Henning, T., \& Beuther, H. 2015, A\&A, 581, A74

Abreu-Vicente, J., Ragan, S., Kainulainen, J., et al. 2016, A\&A, 590, A131

Aladro, R., Martín, S., Riquelme, D., et al. 2015, A\&A, 579, A101

Alstott, J., Bullmore, E., \& Plenz, D. 2014, PLoS One, 9, e85777

Alves, J., Lombardi, M., \& Lada, C. J. 2017, A\&A, 606, L2

Alves de Oliveira, C., Schneider, N., Merín, B., et al. 2014, A\&A, 568, A98

Anderson, L. D., Bania, T. M., Balser, D. S., et al. 2014, ApJS, 212, 1

André, P., Di Francesco, J., Ward-Thompson, D., et al. 2014, in Protostars and Planets VI, eds. H. Beuther, R. S. Klessen, C. P. Dullemond, \& T. Henning (Tucson, AZ: University of Arizona Press), 27

Astropy Collaboration (Price-Whelan, A. M., et al.) 2018, AJ, 156, 123 
Ballesteros-Paredes, J., Vázquez-Semadeni, E., Gazol, A., et al. 2011, MNRAS, 416, 1436

Bertoldi, F., \& McKee, C. F. 1992, ApJ, 395, 140

Bialy, S., Burkhart, B., \& Sternberg, A. 2017, ApJ, 843, 92

Bigiel, F., Leroy, A. K., Jiménez-Donaire, M. J., et al. 2016, ApJ, 822, L26

Burkhart, B., Collins, D. C., \& Lazarian, A. 2015, ApJ, 808, 48

Burkhart, B., Stalpes, K., \& Collins, D. C. 2017, ApJ, 834, L1

Carlhoff, P., Nguyen Luong, Q., Schilke, P., et al. 2013, A\&A, 560, A24

Caselli, P., Walmsley, C. M., Zucconi, A., et al. 2002, ApJ, 565, 344

Chen, H. H.-H., Burkhart, B., Goodman, A., \& Collins, D. C. 2018, ApJ, 859, 162

Churchwell, E., Babler, B. L., Meade, M. R., et al. 2009, PASP, 121, 213

Corbelli, E., Elmegreen, B. G., Braine, J., \& Thilker, D. 2018, A\&A, 617, A125

Dempsey, J. T., Thomas, H. S., \& Currie, M. J. 2013, ApJS, 209, 8

Druard, C., Braine, J., Schuster, K. F., et al. 2014, A\&A, 567, A118

Dumouchel, F., Faure, A., \& Lique, F. 2010, MNRAS, 406, 2488

Dunham, M. K., Rosolowsky, E., Evans, N. J., I., Cyganowski, C., \& Urquhart, J. S. 2011, ApJ, 741, 110

Endres, C. P., Schlemmer, S., Schilke, P., Stutzki, J., \& Müller, H. S. P. 2016, J. Mol. Spectr., 327, 95

Faesi, C. M., Lada, C. J., \& Forbrich, J. 2018, ApJ, 857, 19

Federrath, C., \& Klessen, R. S. 2013, ApJ, 763, 51

Federrath, C., Roman-Duval, J., Klessen, R. S., Schmidt, W., \& Mac Low, M.-M. 2010, A\&A, 512, A81

Feng, S., Beuther, H., Zhang, Q., et al. 2016, A\&A, 592, A21

Fischera, J. 2014, A\&A, 571, A95

Gao, Y., \& Solomon, P. M. 2004, ApJ, 606, 271

Gerner, T., Beuther, H., Semenov, D., et al. 2014, A\&A, 563, A97

Giannetti, A., Wyrowski, F., Brand, J., et al. 2014, A\&A, 570, A65

Ginsburg, A., \& Mirocha, J. 2011, Astrophysics Source Code Library [record ascl:1109.001]

Girichidis, P., Konstandin, L., Whitworth, A. P., \& Klessen, R. S. 2014, ApJ, 781, 91

Goldsmith, P. F., Heyer, M., Narayanan, G., et al. 2008, ApJ, 680, 428

Goodman, A. A., Pineda, J. E., \& Schnee, S. L. 2009, ApJ, 692, 91

Goodman, A. A., Alves, J., Beaumont, C. N., et al. 2014, ApJ, 797, 53

Graninger, D. M., Herbst, E., Öberg, K. I., \& Vasyunin, A. I. 2014, ApJ, 787, 74

Hacar, A., Bosman, A. D., \& van Dishoeck, E. F. 2020, A\&A 635, A4

Herbst, E., Terzieva, R., \& Talbi, D. 2000, MNRAS, 311, 869

Herrera, C. N., Pety, J., Hughes, A., et al. 2020, A\&A, 634, A121

Hughes, A., Meidt, S. E., Colombo, D., et al. 2013, ApJ, 779, 46

Jackson, J. M., Rathborne, J. M., Shah, R. Y., et al. 2006, ApJS, 163, 145

Jackson, J. M., Finn, S. C., Chambers, E. T., Rathborne, J. M., \& Simon, R. 2010, ApJ, 719, L185

Jiménez-Donaire, M. J., Bigiel, F., Leroy, A. K., et al. 2019, ApJ, 880, 127

Kainulainen, J., Beuther, H., Henning, T., \& Plume, R. 2009, A\&A, 508, L35

Kauffmann, J., Goldsmith, P. F., Melnick, G., et al. 2017, A\&A, 605, L5

Klessen, R. S. 2000, ApJ, 535, 869

Körtgen, B., Federrath, C., \& Banerjee, R. 2019, MNRAS, 482, 5233

Kritsuk, A. G., Norman, M. L., \& Wagner, R. 2011, ApJ, 727, L20

Lada, C. J., Forbrich, J., Lombardi, M., \& Alves, J. F. 2012, ApJ, 745, 190

Leroy, A. K., Hughes, A., Schruba, A., et al. 2016, ApJ, 831, 16

Li, G.-X., Urquhart, J. S., Leurini, S., et al. 2016, A\&A, 591, A5

Lin, Y., Liu, H. B., Dale, J. E., et al. 2017, ApJ, 840, 22
Lombardi, M., Lada, C. J., \& Alves, J. 2008, A\&A, 489, 143

Louvet, F., Motte, F., Hennebelle, P., et al. 2014, A\&A, 570, A15

Marsh, K. A., Whitworth, A. P., Lomax, O., et al. 2017, MNRAS, 471, 2730

McKee, C. F., \& Zweibel, E. G. 1992, ApJ, 399, 551

Meier, D. S., Walter, F., Bolatto, A. D., et al. 2015, ApJ, 801, 63

Molinari, S., Swinyard, B., Bally, J., et al. 2010, A\&A, 518, L100

Müller, H. S. P., Thorwirth, S., Roth, D. A., \& Winnewisser, G. 2001, A\&A, 370, L49

Müller, H. S. P., Schlöder, F., Stutzki, J., \& Winnewisser, G. 2005, J. Mol. Struct., 742,215

Myers, P. C. 2015, ApJ, 806, 226

Nishimura, Y., Shimonishi, T., Watanabe, Y., et al. 2016a, ApJ, 829, 94

Nishimura, Y., Shimonishi, T., Watanabe, Y., et al. 2016b, ApJ, 818, 161

Ossenkopf-Okada, V., Csengeri, T., Schneider, N., Federrath, C., \& Klessen, R. S. 2016, A\&A, 590, A104

Ostriker, E. C., Stone, J. M., \& Gammie, C. F. 2001, ApJ, 546, 980

Pandian, J. D., \& Goldsmith, P. F. 2007, ApJ, 669, 435

Pandian, J. D., Menten, K. M., \& Goldsmith, P. F. 2009, ApJ, 706, 1609

Pety, J., Guzmán, V. V., Orkisz, J. H., et al. 2017, A\&A, 599, A98

Ragan, S. E., Henning, T., Tackenberg, J., et al. 2014, A\&A, 568, A73

Riener, M., Kainulainen, J., Beuther, H., et al. 2020, A\&A, 633, A14

Robitaille, T., \& Bressert, E. 2012, APLpy: Astronomical Plotting Library in Python, Astrophysics Source Code Library [record ascl : 1208.017]

Sadavoy, S. I., Di Francesco, J., André, P., et al. 2014, ApJ, 787, L18

Sánchez-Monge, Á., Pandian, J. D., \& Kurtz, S. 2011, ApJ, 739, L9

Schilke, P., Walmsley, C. M., Pineau Des Forets, G., et al. 1992, A\&A, 256, 595

Schinnerer, E., Meidt, S. E., Pety, J., et al. 2013, ApJ, 779, 42

Schneider, N., André, P., Könyves, V., et al. 2013, ApJ, 766, L17

Schneider, N., Bontemps, S., Girichidis, P., et al. 2015a, MNRAS, 453, 41

Schneider, N., Ossenkopf, V., Csengeri, T., et al. 2015b, A\&A, 575, A79

Schneider, N., Bontemps, S., Motte, F., et al. 2016, A\&A, 587, A74

Schuller, F., Menten, K. M., Contreras, Y., et al. 2009, A\&A, 504, 415

Schuller, F., Csengeri, T., Urquhart, J. S., et al. 2017, A\&A, 601, A124

Shirley, Y. L. 2015, PASP, 127, 299

Shu, F. H. 1977, ApJ, 214, 488

Stutz, A. M., \& Kainulainen, J. 2015, A\&A, 577, L6

Sun, Y., Yang, J., Xu, Y., et al. 2020, ApJS, 246, 7

Tremblin, P., Schneider, N., Minier, V., et al. 2014, A\&A, 564, A106

Urquhart, J. S., Morgan, L. K., Figura, C. C., et al. 2011, MNRAS, 418, 1689

Usero, A., Leroy, A. K., Walter, F., et al. 2015, AJ, 150, 115

Wang, K., Testi, L., Ginsburg, A., et al. 2015, MNRAS, 450, 4043

Wang, K., Testi, L., Burkert, A., et al. 2016, ApJS, 226, 9

Wang, Y., Bihr, S., Beuther, H., et al. 2020, A\&A 634, A139

Ward, R. L., Wadsley, J., \& Sills, A. 2014, MNRAS, 445, 1575

Watanabe, Y., Sakai, N., Sorai, K., \& Yamamoto, S. 2014, ApJ, 788, 4

Wilson, T. L., Rohlfs, K., \& Hüttemeister, S. 2013, Tools of Radio Astronomy (Berlin: Springer)

Wong, T., Ladd, E. F., Brisbin, D., et al. 2008, MNRAS, 386, 1069

Yang, B., Stancil, P. C., Balakrishnan, N., \& Forrey, R. C. 2010, ApJ, 718, 1062

Zhang, M., Kainulainen, J., Mattern, M., Fang, M., \& Henning, T. 2019, A\&A, 622, A52

Zucker, C., Battersby, C., \& Goodman, A. 2015, ApJ, 815, 23

Zucker, C., Battersby, C., \& Goodman, A. 2018, ApJ, 864, 153 


\section{Appendix A: Excitation temperature and optical depth maps}
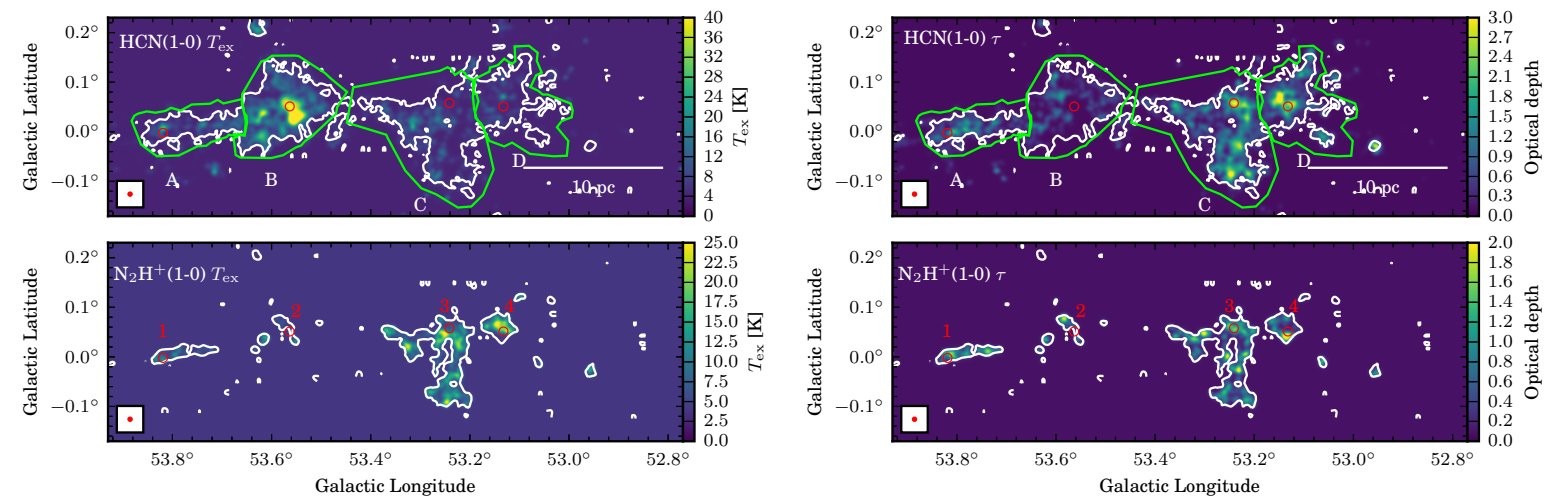

Fig. A.1. Excitation temperature $\left(T_{\mathrm{ex}}\right.$, left $)$ and optical depth $(\tau$, right $)$ maps derived from the HFS fitting after being convolved with a Gaussian FWHM beam of $32^{\prime \prime}$ (Gaussian kernel $\sim 13.59^{\prime \prime}$ ). The thick white contour in each panel traces the $3 \sigma$ level of the respective ${ }^{12} \mathrm{C}$ isotopologue line integrated intensity shown in Fig. 2. The red circles mark the locations whose spectra are shown in Fig. A.2. The beams and polygons are the same as in Fig. 2.
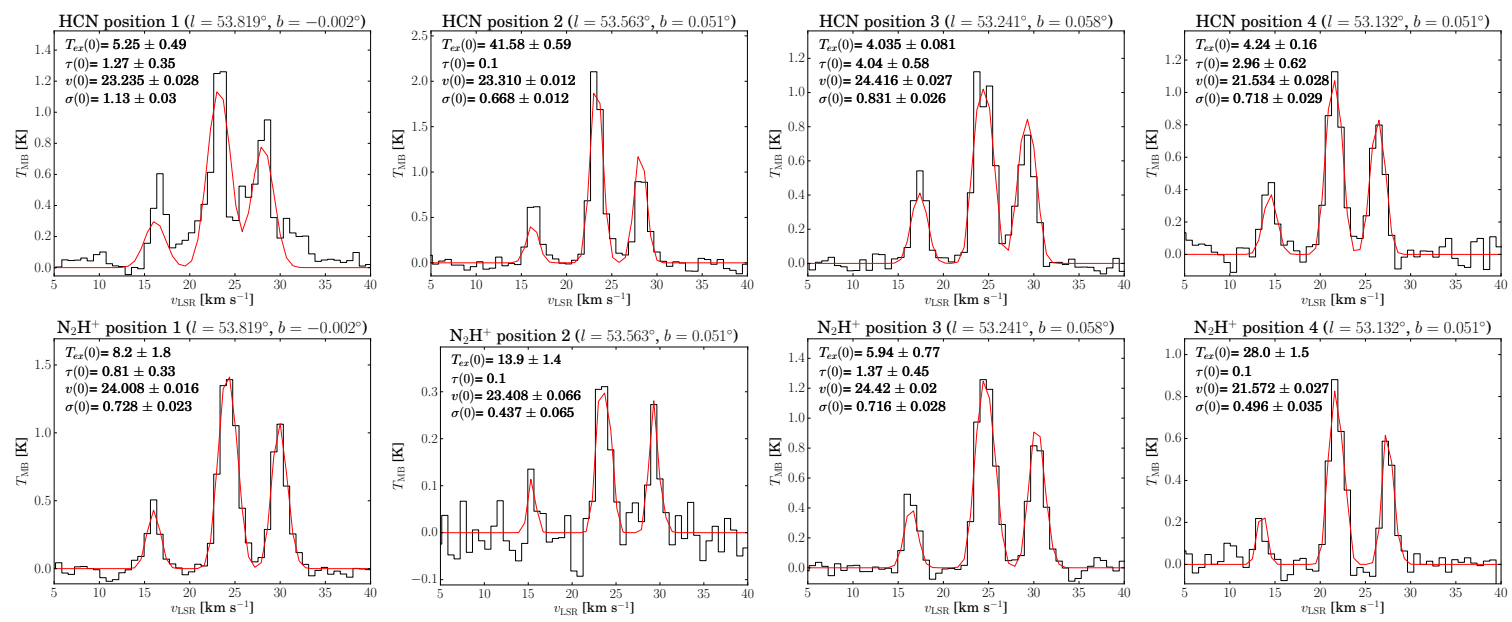

Fig. A.2. Selected $\mathrm{HCN}(1-0)($ top $)$ and $\mathrm{N}_{2} \mathrm{H}^{+}(1-0)$ (bottom) spectra from pixels corresponding to the positions indicated in Fig. A.1. The HFS fits are shown in red. The fitting results, excitation temperature $\left(T_{\text {ex }}\right)$, optical depth $(\tau)$, line center $(v)$, and line width $(\sigma$, velocity dispersion) are listed in each panel.

In this section we show the excitation temperature and optical depth maps derived from the HFS fitting for $\mathrm{HCN}(1-0)$ and $\mathrm{N}_{2} \mathrm{H}^{+}(1-0)$. All four maps are convolved with a Gaussian FWHM beam of $32^{\prime \prime}$ (Gaussian kernel $13.59^{\prime \prime}$ ) in Fig. A.1 and four selected spectra of each transition to show the HFS fitting results (Fig. A.2). We also show the $\mathrm{H}^{13} \mathrm{CO}^{+}(1-0)$ and $\mathrm{HN}^{13} \mathrm{C}(1-0)$ integrated intensity maps in Fig. A.3.

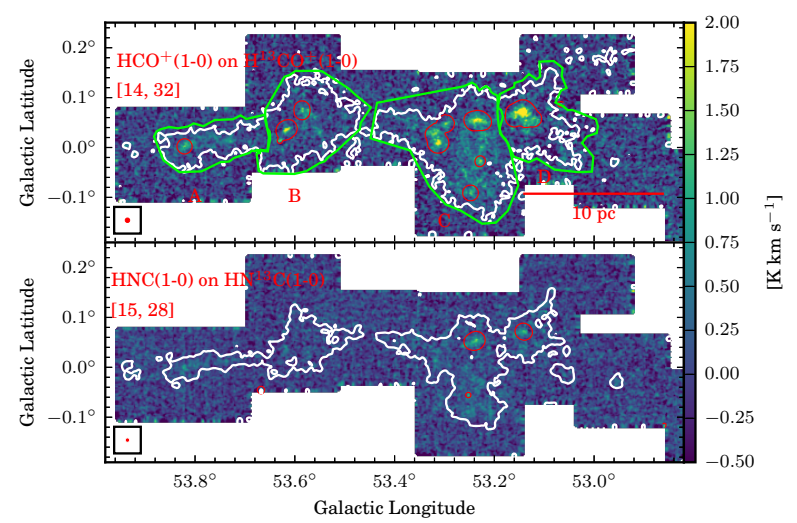

Fig. A.3. $\mathrm{H}^{13} \mathrm{CO}^{+}(1-0)$ and $\mathrm{HN}^{13} \mathrm{C}(1-0)$ integrated intensity maps (color scale and red contours). The red contours outline the area where the ${ }^{13} \mathrm{C}$ lines have a good signal-to-noise ratio and the optical depth correction was applied for the column density calculation. The thick white contour in each panel traces the $3 \sigma$ level of the respective ${ }^{12} \mathrm{C}$ isotopologue line integrated intensity shown in Fig. 2. The beams, ellipses, and polygons are the same as in Fig. 2. 


\section{Appendix B: N-PDFs with optically thin assumption}
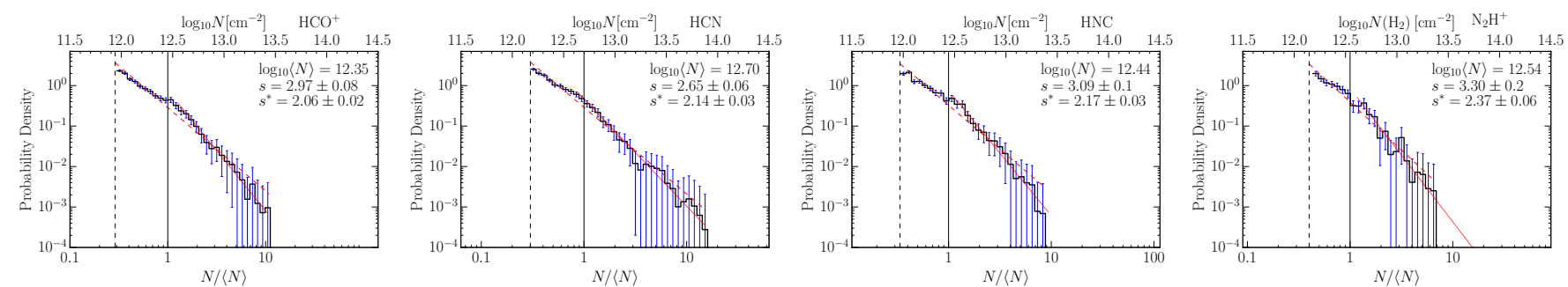

Fig. B.1. N-PDFs of column density derived from the $\mathrm{HCO}^{+}(1-0), \mathrm{HCN}(1-0), \mathrm{HNC}(1-0)$, and $\mathrm{N}_{2} \mathrm{H}^{+}(1-0)$ assuming optically thin emission for the whole filament. We listed in each panel the mean column density $\langle N\rangle$, the power-law index $s$ (the optimized fit, solid line).

\section{Appendix C: Density profile of a singular polytropic cylinder}

This section is adopted from the appendix in Schneider et al. (2016). The N-PDF is defined as the area fraction of gas $A$ with column density $N, p_{N} \propto \mathrm{d} A / \mathrm{d} N$. In this paper, we calculate the probability distribution $p$ of the normalized column density $\log _{10}(N /\langle N\rangle)$, therefore $p \propto \mathrm{d} A / \mathrm{d}\left(\log _{10}(N /\langle N\rangle)\right) \propto N p_{N}$. For a singular polytropic cylinder, the density profile from the axis of the cylinder filament is $\rho(r) \propto r^{-\alpha_{\mathrm{f}}}$. The column density $N$ is an integral of the density, hence $N \propto \rho r \propto r^{-\alpha_{\mathrm{f}}+1}$. The area fraction $A$ is proportional to the cylinder length $z$ and radius $r, A \propto z r \propto r$, therefore

$p_{N} \propto \mathrm{d} A / \mathrm{d} N \propto 1 / r^{-\alpha_{\mathrm{f}}} \propto N^{\alpha_{\mathrm{f}} /\left(1-\alpha_{\mathrm{f}}\right)}$ $p \propto N p_{N} \propto N^{1 /\left(1-\alpha_{\mathrm{f}}\right)}$.

The power-law index of the N-PDF is $s\left(p \propto(N /\langle N\rangle)^{-s}\right)$, hence $\alpha_{\mathrm{f}}=1+1 / s$.

Similarly for a spherical core or clump with a density profile of $\rho(r) \propto r^{-\alpha_{\mathrm{c}}}$, column density $N \propto \rho r \propto r^{-\alpha_{\mathrm{c}}+1}$. In this case, they are $A \propto r^{2}$. Therefore,

$p_{N} \propto \mathrm{d} A / \mathrm{d} N \propto r / r^{-\alpha_{\mathrm{c}}} \propto N^{1+\alpha_{\mathrm{c}} /\left(1-\alpha_{\mathrm{c}}\right)}$

$p \propto N p_{N} \propto N^{2 /\left(1-\alpha_{\mathrm{c}}\right)}$,

and $\alpha_{\mathrm{c}}=1+2 / s$.

Similar calculations are also discussed by Federrath \& Klessen (2013), Fischera (2014), and Myers (2015). 